\title{
FORMULACIÓN DE ELEMENTOS FINITOS VIGA-COLUMNA CON DISCONTINUIDADES EMBEBIDAS PARA MODELAR DAÑO EN ELEMENTOS PRISMÁTICOS DE CONCRETO REFORZADO
}

\author{
Gelacio Juárez Luna $^{(1)}$ y Enrique Tenorio Montero $^{(2)}$
}

\begin{abstract}
RESUMEN
Se formulan elementos finitos viga-columna con discontinuidades interiores para modelar el desarrollo de articulaciones. En estos elementos, el daño se modela como el desarrollo de rótulas, dislocaciones transversales y discontinuidades axiales. Estos elementos finitos utilizan modelos constitutivos para considerar la capacidad de momento, fuerza cortante y fuerza axial, basados en la mecánica del daño y en pruebas experimentales reportadas en la literatura, las cuales incluyen el comportamiento constitutivo del concreto con acero de refuerzo. Para mostrar la capacidad de modelar el daño de los elementos finitos desarrollados, se presentan ejemplos numéricos de vigas y marcos de concreto reforzados sujetos a cargas que inducen daño. En estos ejemplos numéricos, las curvas de la carga contra el desplazamiento calculadas son congruentes con las reportadas en la literatura.
\end{abstract}

Palabras clave: colapso; discontinuidades; daño; dislocación; articulación; ablandamiento

\section{BEAM-COLUMN FINITE ELEMENT WITH EMBEDDED DISCONTINUITIES FOR MODELLING DAMAGE IN REINFORCED CONCRETE PRISMATIC ELEMENTS}

\begin{abstract}
Frame elements with embedded discontinuities to model the development of hinges are formulated. In these elements, damage is modelled as the development of hinges, transversal dislocations and axial discontinuities. These elements use constitutive models to include the capacity of the moment, shear force and axial force, based on the damage mechanics and experimental tests reported in the literature, which include the constitutive behaviour of concrete with steel reinforcement bars. To show the ability of the formulated finite elements with embedded discontinuities for modelling damage, numerical examples of reinforced concrete structural beams and frames under loads, which induce damage. In these numerical examples, the load-displacement curves are in agreement with those reported in the literature.
\end{abstract}

Keywords: collapse; discontinuities; damage; dislocation; articulation; softening

Recibido el 9 de junio de 2016, Revisado el 8 de junio de 2017, Aprobado el 18 de junio de 2017. Se aceptarán comentarios y/o discusiones hasta cinco meses después de su publicación.

(1) Departamento de Materiales, Universidad Autónoma Metropolitana, San Pablo 180, Col. Reynosa-Tamaulipas, Del. Azcapotzalco, 02200, México, D.F. Teléfono: (55) 5318-9085; Fax: (55)5318-9085; gjl@azc.uam.mx

(2) Dirección General de Ingeniería y Arquitectura, Universidad Tecnológica de Panamá, Avenida Universidad Tecnológica de Panamá, Campus Metropolitano Víctor Levi Sasso, Panamá, Ciudad de Panamá, Teléfono: (507) 6316-6152; Fax:290-8400; etenorio14@hotmail.com 


\section{INTRODUCCIÓN}

Una estructura presenta daño incipiente cuando las acciones de fuerza que actúan sobre ella producen que los materiales alcancen sus valores umbrales de resistencia. El incremento o acumulación de daño en los materiales puede producir el colapso de las estructuras. El daño puede ocurrir cuando se presenta inestabilidad elástica (pandeo), excesiva deformación plástica (fluencia generalizada), fatiga (cargas cíclicas), corrosión, fractura, etc. El estudio del inicio del daño y la evolución al colapso de estructuras es de importancia para conocer la carga última o la capacidad residual, lo cual depende del comportamiento constitutivo de los materiales, así como de las acciones de fuerza que actúan en las estructuras.

En estructuras como los edificios, sus elementos estructurales pueden ser afectados por cargas o por acciones externas tales como: sismos, explosiones, colapso de estructuras vecinas, actividades de construcción, etc. El método de los elementos finitos es el método numérico más utilizado para estudiar el inicio del daño y la evolución al colapso de estructuras, en el que los elementos estructurales se discretizan con elementos sólidos o viga-columna. A estos elementos se les asignan los comportamientos constitutivos de los materiales, que pueden incluir comportamientos no lineales. Para el caso en que las estructuras se discretizan con elementos viga-columna, Baker y Hyman (1969) modelaron el daño en elementos vigacolumna como articulaciones plásticas, utilizando modelos constitutivos momento-curvatura elastoplásticos o con una pendiente reducida, esta aproximación no consideran el ablandamiento que se presenta después de alcanzar la carga última. Otra aproximación fue desarrollados por Ehrlich y Armero (2004), quienes formularon elementos finitos tipo viga de Timoshenko con discontinuidades embebidas, los cuales tienen la capacidad de modelar discontinuidades de rotación (articulación) y desplazamiento transversal (desgarramiento); sin embargo, estos elementos requieren de funciones definidas como operadores de deformación para mejorar la solución. La formulación de estos elementos proporcionan matrices de rigideces asimétricas que pueden presentan dificultades numéricas en el proceso de cálculo. Posteriormente, Armero y Ehrlich (2006) desarrollaron un elemento finito con discontinuidades embebidas con base en la teoría de vigas de Euler-Bernoulli, el cual sólo considera la discontinuidad de rotación. Dujc et al. (2010) desarrollaron un modelo multi-escala para el estudio de discontinuidades interiores axiales y rotacionales, en el cual utilizaron elementos sólidos en 2D para la micro-escala y elementos vigas Euler-Bernoulli para la macro-escala. Juárez y Ayala (2012) desarrollaron unas formulaciones para vigas gruesas y delgadas con discontinuidades embebidas con base en funcionales de energía, cuyas aproximaciones con elementos finitos proporcionan matrices simétricas, bien condicionadas, las cuales fueron validadas con ejemplos reportados en la literatura. Estos autores desarrollaron una formulación de modelos constitutivos por flexión y cortante, considerando que existe ablandamiento después de alcanzar un valor umbral. Jukic et al. (2013) formularon un elemento finito con discontinuidades interiores basado en la teoría de Euler-Bernoulli que sólo modela la discontinuidad de la rotación. El comportamiento constitutivo del momento contra la curvatura para las vigas de concreto reforzado es semejante al desarrollado por Dujc et al. (2010), en el que ocurre ablandamiento posterior al intervalo plástico. Jukic (2013) desarrolló un elemento finito que considera la no-linealidad axial en vigas tipo Euler-Bernoulli y Timoshenko, mediante una sección de viga discretizada en fibras, a las que se les asigna un comportamiento constitutivo esfuerzo-deformación. Estas fibras son esforzadas axialmente en la sección transversal de la viga debido a fuerzas producidas por la acción de los momentos flexionantes.

En este trabajo se desarrolla un elemento viga-columna delgado, con base en la teoría de EulerBernoulli, que tiene la capacidad de modelar discontinuidades en la rotación y en el desplazamiento axial y otro elemento grueso, con base en la teoría de Timoshenko, que además es capaz de modelar la discontinuidad del desplazamiento transversal. Estos elementos finitos se formulan a partir de funcionales de energía de barras y vigas, respectivamente. A diferencia de los trabajos de Juárez (2006) y Juárez y Ayala (2012) que obtienen las matrices de rigideces independientes de los elementos barras y los elementos vigas con discontinuidades interiores, en este trabajo las matrices de rigideces se acoplan para tener los elementos 
viga-columna, los cuales tiene la capacidad de desarrollar discontinuidad en los desplazamientos axiales, en la rotación y en los desplazamientos transversales. Se presentan ejemplos de aplicación numérica que muestran la capacidad de estos elementos para modelar el proceso de falla en estructuras.

\section{FORMULACIÓN DE ELEMENTOS VIGA-COLUMNA CON DISCONTINUIDADES INTERIORES}

\section{Elemento barra con discontinuidades interiores}

Sea una barra con longitud $L$, sección transversal $A$ y módulo elástico $E$, como se muestra en la Figura 1a, la cual se somete a carga axial, $f$, hasta que ocurre una concentración de deformaciones en la zona $S$, donde se presenta una discontinuidad o salto en el desplazamiento axial, [|u|], por lo que el dominio de la barra $\Omega I[0, \mathrm{~L}]$, queda dividido por la discontinuidad $S$, tal que $\Omega=\Omega^{-}+\Omega^{+}$.
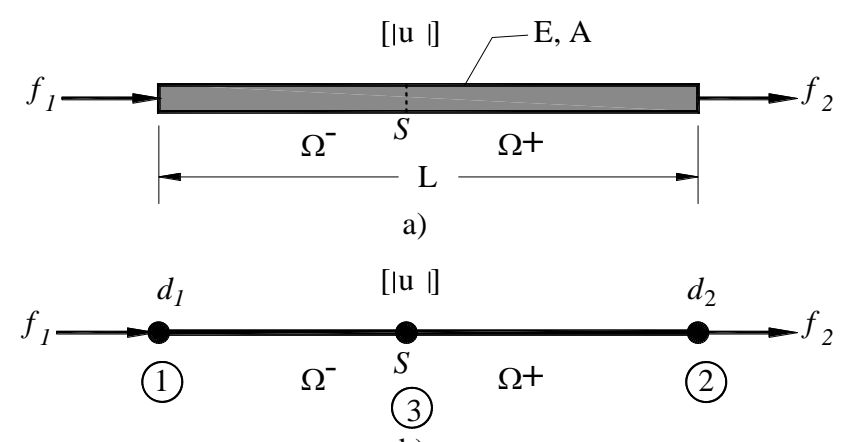

b)

Figura 1. Elemento barra: a) con discontinuidad interior y b) discretización con un elemento finito

El funcional de energía del elemento barra con discontinuidad axial es (Juárez 2006):

$\Pi(u,[|u|])=\int_{\Omega}[W(\bar{\varepsilon})-b \cdot u] d \Omega-f_{i} \cdot u_{i}+\int_{0}^{[|u|]} T_{S} \cdot[|u|] d[|u|]$

donde $u$ es el desplazamiento axial, $W(\bar{\varepsilon})=\int_{0}^{\bar{\varepsilon}} \sigma(\bar{\varepsilon}) d \bar{\varepsilon}$ es la densidad de energía de deformación, $b$ es la fuerza de cuerpo, $f_{i} \cdot u_{i}$ es el trabajo externo debido a las cargas concentradas, y $\int_{0}^{[|u|]} T_{S} \cdot[|u|] d[|u|]$ es el trabajo en la discontinuidad debida a las tracciones, $T_{s}$.

La aproximación con elementos finitos del desplazamiento, $u(\mathbf{x})$, con discontinuidad embebida, $[|u|]$, de este elemento barra es:

$u(\mathbf{x})=N(\mathbf{x}) d+M_{s}(\mathbf{x})[|u|]$

donde $d$ son los desplazamientos en los nodos de extremos del elemento y $[|u|]$ es el salto de desplazamiento axial en el nodo interno, como se muestra en la Figura 1b. En la ec. (2), $N(\mathbf{x})$ contiene las funciones de forma estándar definidas como: 


$$
\begin{aligned}
& N(\mathbf{x})=\left[\begin{array}{ll}
N_{1}(x) & N_{2}(x)
\end{array}\right] \\
& N_{1}(x)=\frac{x_{2}-x}{L}, N_{2}(x)=\frac{x-x_{1}}{L}
\end{aligned}
$$

La función $M_{s}(\mathbf{x})$, mostrada en la Figura 2, que aproxima el salto de desplazamiento se define como (Oliver, 1996):

$$
M_{S}(\mathbf{x})=H_{S}(\mathbf{x})-\varphi(\mathbf{x})
$$

en la que $H_{s}(\mathbf{x})$ es la función salto de Heaviside y $\varphi(\mathbf{x})$ es una función continua tal que:

$$
\begin{array}{ll}
\varphi(\mathbf{x})=0 & \forall \mathbf{x} \in \Omega^{-} \\
\varphi(\mathbf{x})=1 & \forall \mathbf{x} \in \Omega^{+}
\end{array}
$$
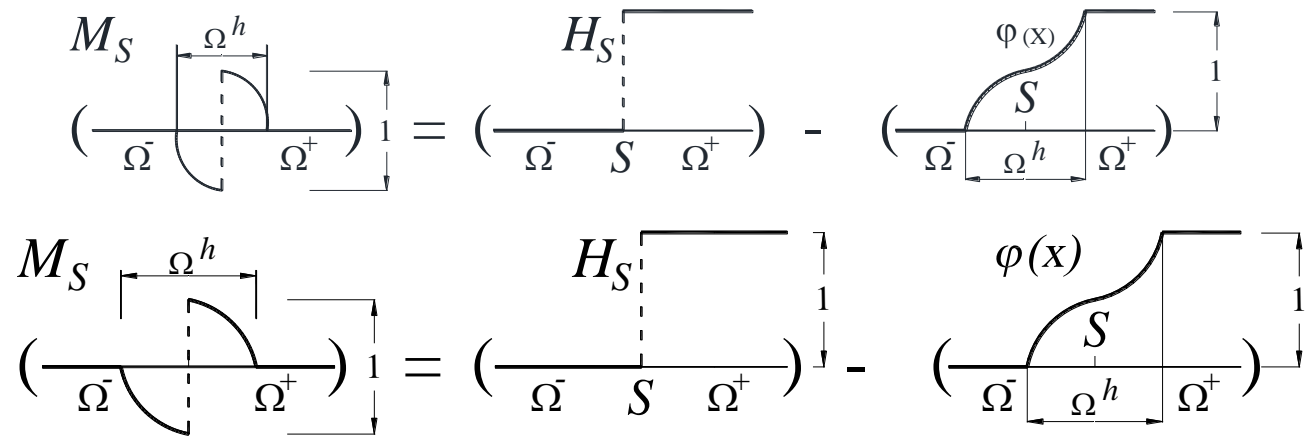

Figura 2. Representación gráfica de la función $M_{S}$

Es de interés mencionar que la ubicación de la discontinuidad en $S$ en la Figura 2, sólo indica que está dentro del elemento, pero no necesariamente en el centro. Estos elementos, llamados con discontinuidades embebidas, tienen las discontinuidades $[|\mathrm{u}|],[|\theta|],[|\mathrm{w}|]$ (saltos) embebidos y distribuidos en el elemento mediante la función $M_{s}$ de la ec. (4).

Las deformaciones continuas se aproximan como:

$\bar{\varepsilon}(\mathbf{x})=B(\mathbf{x}) \cdot d+B_{C}(\mathbf{x}) \cdot[|u|]$

donde las derivadas de las funciones de forma definidas en las ecs. (3) y (4), respectivamente, son:

$$
\begin{aligned}
& B(\mathbf{x})=\frac{1}{L}\left[\begin{array}{ll}
-1 & 1
\end{array}\right] \\
& B_{C}(\mathbf{x})=-\frac{1}{L}
\end{aligned}
$$

Sustituyendo las ecs. (2) y (6) en la ec. (1), se obtiene: 


$$
\begin{aligned}
& \Pi(d,[|u|))=\int_{\Omega}\left[C\left(d^{T} B^{T}+[|u|]^{T} B_{C}^{T}\right) \cdot\left(B d+B_{C}[|u|]\right)-b \cdot\left(d^{T} N^{T}(x)+[|u|]^{T} N_{C}^{T}\right)\right] \cdot d \Omega \\
& -\left(d^{T} N^{T}(x)+[|u|]^{T} N_{C}^{T}\right) F_{i}+\int_{0}^{u} \int_{0}^{u} T_{S}([|u|]) d[|u|]
\end{aligned}
$$

Para optimizar el funcional de la ec. (8), éste se deriva respecto a $d$ y $[|u|]$ e iguala respectivamente a cero, obteniéndose dos ecuaciones que posteriormente se linealizan con series de Taylor tal que:

$$
\left[\begin{array}{cc}
\int_{V} B^{T} C B \cdot d v & \int_{V} B^{T} C B_{C} \cdot d v \\
\int_{V} B_{C}^{T} C B \cdot d v & \int_{V} B_{C}^{T} C B_{C} \cdot d v+\int_{\Gamma} C_{N}^{T} \cdot d \Gamma
\end{array}\right] \cdot\left[\begin{array}{c}
\Delta d \\
\Delta[|u|]
\end{array}\right]=\left[\begin{array}{c}
\Delta f_{\text {ext }} \\
\Delta f_{\text {int }}
\end{array}\right]
$$

Sustituyendo los términos de la ec. (7) en la ec. (9) se obtiene la siguiente matriz de rigidez de una barra con discontinuidad axial:

$$
\left[\begin{array}{ccc}
\frac{A E}{L} & \frac{-A E}{L} & \frac{A E}{L} \\
\frac{-A E}{L} & \frac{A E}{L} & \frac{-A E}{L} \\
\frac{A E}{L} & \frac{-A E}{L} & \frac{A E}{L}+A C_{N}^{T}
\end{array}\right]^{(n-1)} \cdot\left\{\begin{array}{c}
\Delta d_{1} \\
\Delta d_{2} \\
\Delta[|u|]
\end{array}\right\}^{(n)}=\left\{\begin{array}{l}
f_{1} \\
f_{2} \\
f_{S}
\end{array}\right\}^{(n-1)}
$$

donde $C_{N}^{T}=\partial T_{S} / \partial[|u|]$ es el operador constitutivo tangente en la discontinuidad axial. El vector de fuerzas residuales en los nodos y en la discontinuidad, para cada paso de carga $n$ se define, respectivamente, como:

$$
\begin{aligned}
& {\left[\begin{array}{l}
f_{1} \\
f_{2}
\end{array}\right]^{(n-1)}=\left[\begin{array}{l}
F_{\text {ext }} \\
F_{\text {ext } 2}
\end{array}\right]^{(n)}-\frac{A E}{L}\left[\left[\begin{array}{cc}
1 & -1 \\
-1 & 1
\end{array}\right] \cdot\left[\begin{array}{l}
d_{1} \\
d_{2}
\end{array}\right]^{(n-1)}+\left[\begin{array}{c}
1 \\
-1
\end{array}\right] \cdot[|u|]^{(n-1)}\right]} \\
& f_{S}^{(n-1)}=\frac{A E}{L}\left[\left[\begin{array}{ll}
-1 & 1
\end{array}\right]+[|u|]^{(n-1)}\right]+A T_{S}[|u|]^{(n-1)}
\end{aligned}
$$

\section{Viga delgada con discontinuidades interiores}

Se consideran como vigas delgadas aquellas cuya relación peralte, $h$, entre longitud $L$, sea $h / L \leq 0.2$, de otra forma se considerarán como vigas gruesas. En la Figura 3 se muestra una viga delgada sujeta a una carga distribuida $q(x)$, momento $M$ y fuerzas transversales $V$ en sus extremos. La viga tiene una la longitud, $L$, momento de inercia, $I$, y módulo elástico, $E$. En las vigas delgadas sólo pueden ocurrir articulaciones debidas a discontinuidades en el campo de las rotaciones $[|\theta|]$. 


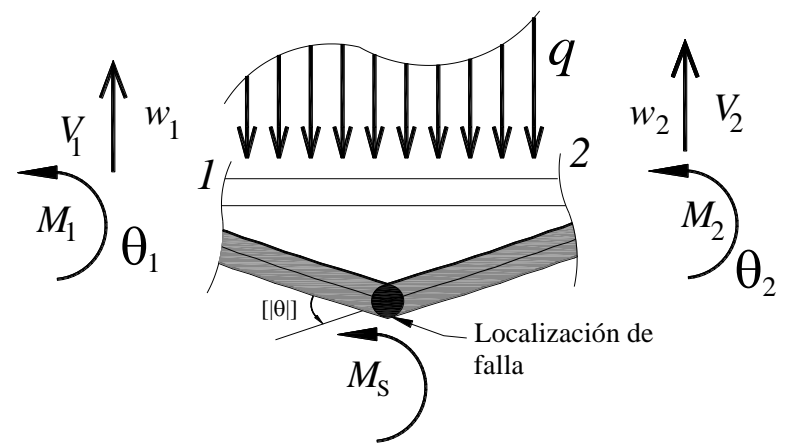

Figura 3. Elemento viga delgada con discontinuidad en rotación

El funcional de energía, desarrollado por Juárez y Ayala (2012), para la viga delgada con discontinuidades es:

$\Pi(w,[|\theta|])=\int_{L}\left(\psi^{M}\left(\bar{\kappa}^{w}\right)-q(x) \cdot w\right) d x+\int_{S} \psi^{M_{S}}([|\theta|]) d s-\left.M^{*} \cdot \theta\right|_{\Gamma}-\left.V^{*} \cdot w\right|_{\Gamma}$

donde $\psi^{M}$ corresponde a la densidad de energía a flexión, dependiente de la curvatura continua, $\bar{\kappa}$, como se muestra en la Figura 4a. La densidad de energía de deformación, $\psi^{M_{s}}$, liberada debido a la formación de una articulación depende de $[|\theta|]$, como se muestra en la Figura 4b. Los momentos y los cortantes prescritos en los extremos son, respectivamente, $M^{*}$ y $V^{*}$. La ec. (13) se reescribe como:

$$
\begin{aligned}
\Pi(w,[|\theta|])= & \int_{L} \frac{1}{2}\left[\frac{\partial^{2} w(x)}{\partial x^{2}}-\frac{\partial \varphi(x)[|\theta|]}{\partial x}\right]^{T} E I\left[\frac{\partial^{2} w(x)}{\partial x^{2}}-\frac{\partial \varphi(x)[|\theta|]}{\partial x}\right] d x-\int_{L} q(x) w(x) d x+\int_{S} M[|\theta|] d S \\
& -\left.M^{*} \cdot \theta\right|_{\Gamma}-\left.V^{*} \cdot w\right|_{\Gamma}
\end{aligned}
$$

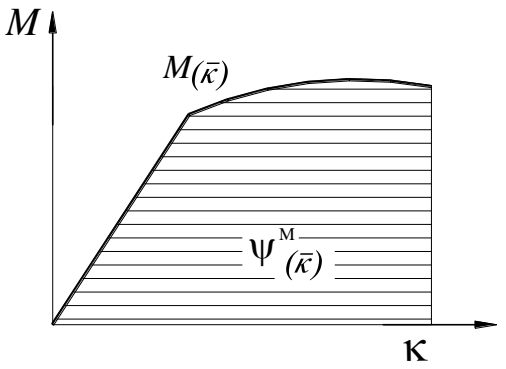

a)

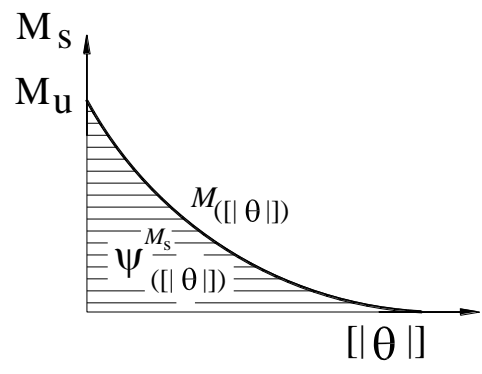

b)

Figura 4. Densidad de energía de deformación en: a) en la parte continua de la viga y b) en la zona de localización (tomada de Juárez y Ayala 2012)

Los desplazamientos transversales regulares, $w(x)$, se interpolan como:

$w(x)=N(x) d$

donde $d$ es el vector de desplazamientos en los nodos y $N(x)$ son las funciones de forma, que en el caso de vigas delgadas. 


$$
N_{1}=\frac{1}{L^{3}}\left(2 x^{3}-3 L x^{2}+L^{3}\right), N_{2}=\frac{1}{L^{3}}\left(x^{3} L-2 x^{2}-2 x^{2} L^{2}+L^{3} x\right), N_{3}=\frac{1}{L^{3}}\left(3 L x^{2}-2 x^{2}\right), N_{4}=\frac{1}{L^{3}}\left(L x^{3}-L^{2} x^{2}\right)
$$

Sustituyendo la ec. (15) en la ec. (14) se obtiene:

$$
\begin{aligned}
\Pi(d,[|\theta|])= & \int_{0}^{L} \frac{1}{2}\left[\frac{\partial^{2} N(x) d}{\partial x^{2}}-\frac{\partial \varphi(x)[|\theta|]]^{T}}{\partial x}\right]^{T}\left[\frac{\partial^{2} N(x) d}{\partial x^{2}}-\frac{\partial \varphi(x)[|\theta|]}{\partial x}\right] d x \\
& -\int_{0}^{L} d^{T} N^{T} q(x) d x+\int_{S} \int_{0}^{[|\theta|]} M[|\theta|] d[|\theta|] d S-\left.M^{*} \cdot N(x) d\right|_{\Gamma}-\left.V^{*} \cdot N(x) d\right|_{\Gamma}
\end{aligned}
$$

La función $\varphi(x)$ está dada por:

$$
\varphi(x)=\frac{x}{L}
$$

Con las derivadas de las funciones de forma definidas por las ecs. (16) y (18), se determinan $B(x)$ y $B_{C}(x)$, respectivamente, como:

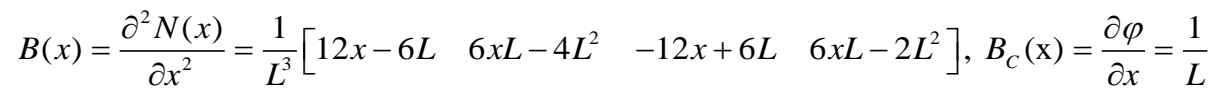

Sustituyendo las ec. (19) en la ec. (17) se obtiene:

$$
\begin{aligned}
\Pi(d,[|\theta|])= & \int_{0}^{L} \frac{1}{2}\left[B(x) d-B_{C}(x)[|\theta|]\right]^{T} E I\left[B(x) d-B_{C}(x)[|\theta|]\right] d x-\int_{0}^{L} d^{T} N^{T}(x) q(x) d x \\
& +\int_{S} \int_{0}^{\theta} M([|\theta|]) d[|\theta|] d S-\left.d^{T} N(x)^{T} \cdot M^{*}\right|_{\Gamma}-d^{T} N(x)^{T} \cdot V_{\Gamma}^{*}
\end{aligned}
$$

Para optimizar el funcional de la ec. (20), éste se deriva respecto a $d$ y $[|\theta|]$, respectivamente. Posteriormente estas dos ecuaciones no lineales se igualan a cero, las cuales se linealizan con series de Taylor, tal que:

$$
\begin{aligned}
& \int_{0}^{L} B(x)^{T} E I B(x) \Delta d \cdot d x-\int_{0}^{L} B(x)^{T} E I B_{C}(x) \Delta[|\theta|] \cdot d x+\int_{0}^{L} B(x)^{T} \sigma\left(B(x) d-B_{C}(x)[|\theta|]\right) \cdot d x-F_{\text {ext }}=0 \\
& \int_{0}^{L} B_{C}{ }^{T} E I B(x) \Delta d \cdot d x-\int_{0}^{L} B_{C}{ }^{T} E I B_{C}(x) \Delta[|\theta|]+\int_{0}^{L} B_{C}^{T}(x) \sigma\left(B(x) d-B_{C}(x)[|\theta|]\right) d x+M(\theta)+I C_{b}^{T} \Delta[|\theta|]=0
\end{aligned}
$$

donde $C_{b}^{T}=\partial M_{[\theta \theta]} / \partial[|\theta|]$ es el operador constitutivo tangente del momento contra el salto de rotación, los dos primeros términos de cada expresión representan las rigideces del elemento, los términos restantes representas los residuos de fuerzas y momentos. Organizando los términos de la ec. (21) en una matriz y vectores se tiene:

$$
\left[\begin{array}{cc}
\int_{0}^{L} B(x)^{T} E I B(x) d x & \int_{0}^{L} B(x)^{T} E I B_{C} d x \\
B_{C}{ }^{T} E I B(x) d x & \int_{0}^{L} B_{C}{ }^{T} E I B_{C} d x+C_{b}^{T} I
\end{array}\right]\left[\begin{array}{c}
\Delta d \\
\Delta[|\theta|]
\end{array}\right]=\left[\begin{array}{c}
f_{a} \\
f_{\mathrm{i}}
\end{array}\right]
$$


Sustituyendo la ec. (19) en la ec. (22) e integrando, se obtiene la matriz de rigidez del elemento viga delgada con discontinuidad en la rotación:

$\left[\begin{array}{ccccc}\frac{12 E I}{L^{3}} & \frac{6 E I}{L^{2}} & -\frac{12 E I}{L^{3}} & \frac{6 E I}{L^{2}} & 0 \\ \frac{6 E I}{L^{2}} & \frac{4 E I}{L} & -\frac{6 E I}{L^{2}} & \frac{2 E I}{L} & \frac{E I}{L} \\ -\frac{12 E I}{L^{3}} & -\frac{6 E I}{L^{2}} & \frac{12 E I}{L^{3}} & -\frac{6 E I}{L^{2}} & 0 \\ \frac{6 E I}{L^{2}} & \frac{2 E I}{L} & -\frac{6 E I}{L^{2}} & \frac{4 E I}{L} & -\frac{E I}{L} \\ 0 & \frac{E I}{L} & 0 & -\frac{E I}{L} & \frac{E I}{L}+I C_{b}^{T}\end{array}\right]^{(n-1)}\left[\begin{array}{c}\Delta w_{1} \\ \Delta \theta_{1} \\ \Delta w_{2} \\ \Delta \theta_{2} \\ \Delta[|\theta|]\end{array}\right]^{n}=\left[\begin{array}{c}f_{1} \\ m_{1} \\ f_{2} \\ m_{2} \\ m_{S}\end{array}\right]^{n-1}$

donde los términos del vector de los residuos para cada incremento, $n$, de desplazamiento o carga son:

$$
\begin{aligned}
& {\left[\begin{array}{c}
f_{1} \\
m_{1} \\
f_{2} \\
m_{2}
\end{array}\right]^{(n-1)}=\left[\begin{array}{c}
f_{\text {lext }} \\
M_{\text {lext }} \\
f_{\text {2ext }} \\
M_{2 \text { 2ext }}
\end{array}\right]^{(n)}-\frac{E I}{L^{3}}\left[\begin{array}{cccc}
12 & 6 L & -12 & 6 L \\
6 L & 4 L^{2} & -6 L & 2 L^{2} \\
-12 & -6 L & 12 & -6 L \\
6 L & 2 L^{2} & 6 L & 4 L^{2}
\end{array}\right]\left[\begin{array}{l}
w_{1} \\
\theta_{1} \\
w_{2} \\
\theta_{2}
\end{array}\right]^{(n-1)}+\frac{E I}{L}\left[\begin{array}{l}
0 \\
1 \\
0 \\
1
\end{array}\right][|\theta|]^{n-1}} \\
& m_{S}^{(n-1)}=-\frac{E I}{L}\left[\begin{array}{llll}
0 & 1 & 0 & 1
\end{array}\right]\left[\begin{array}{l}
w_{1} \\
\theta_{1} \\
w_{2} \\
\theta_{2}
\end{array}\right]^{(n-1)}+\frac{E I}{L}[|\theta|]-M([|\theta|])^{n-1}
\end{aligned}
$$

\section{Viga gruesa con discontinuidades interiores}

En vigas gruesas, el desplazamiento transversal, $w, \mathrm{y}$ los giros $\theta$ son independientes, por lo que este elemento puede presentar discontinuidades en el desplazamiento transversal, [ $|w|]$, y en la rotación, $[|\theta|]$, como se muestra en la Figura 5. Sus propiedades mecánicas y geométricas son: el módulo elástico $E$, momento de inercia $I$, longitud $L$ y sección transversal de área efectiva a cortante $A_{s}$.

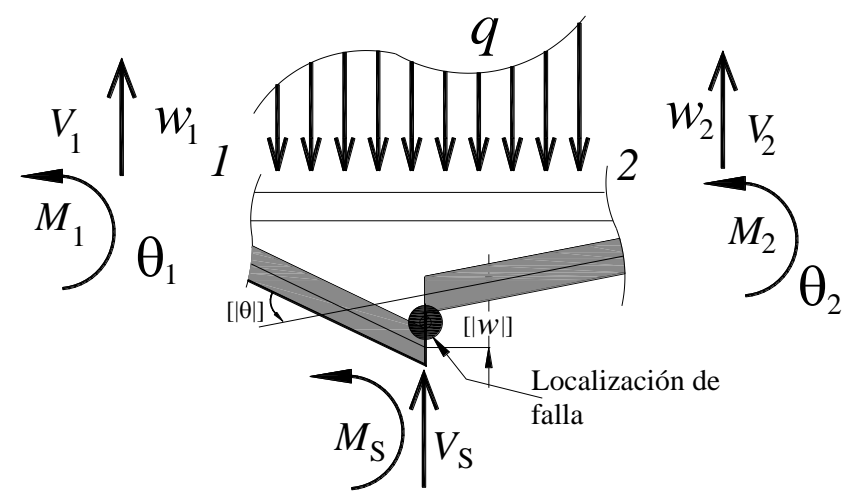

Figura 5. Elemento viga gruesa con discontinuidad en desplazamiento transversal y rotación

El funcional de energía del elemento viga gruesa con discontinuidades, desarrollado por Juárez y Ayala (2012), es: 
$\Pi(w, \theta,[|w|],[|\theta|])=\int_{L}\left[\frac{1}{2}\left(\frac{\partial \bar{\theta}}{\partial x}\right)^{T} E I\left(\frac{\partial \bar{\theta}}{\partial x}\right)+\frac{1}{2}\left(\frac{\partial \bar{w}}{\partial x}-\bar{\theta}\right)^{T} \alpha\left(\frac{\partial \bar{w}}{\partial x}-\bar{\theta}\right)+q w\right] d x+\int_{\Gamma} M_{S}([|\theta|]) d \Gamma+\int_{\Gamma} V_{S}([|w|]) d \Gamma$

en la que $V_{S}$ y $M_{S}$ son respectivamente el cortante y momento en la zona de la discontinuidad, $\bar{w}$ y $\bar{\theta}$ son los desplazamientos y rotaciones continuas que se aproximan respectivamente como:

$\bar{\theta}(x)=\theta(x)-\varphi(x) \cdot[|\theta|], \overline{\mathrm{w}}(x)=w(x)-\varphi(x) \cdot[|w|]$

Puesto que los campos de rotación y desplazamientos son independientes, éstos se aproximan respectivamente como:

$\theta(x)=N_{\theta}(x) \cdot \theta, \quad w(x)=N_{w}(x) \cdot w$

Sustituyendo la ec. (28) en la ec. (27) y posteriormente en la ec. (26), se tiene:

$\Pi(w, \theta,[|w|],[|\theta|])=\int_{0}^{L} \frac{1}{2}\left[\frac{\partial\left(N_{\theta}(x) \cdot \theta-\varphi(x) \cdot[|\theta|]\right)}{\partial x}\right]^{T} E I\left[\frac{\partial\left(N_{\theta}(x) \cdot \theta-\varphi(x) \cdot\right)[|\theta|]}{\partial x}\right] d x-\int_{0}^{L}\left(N_{w}(x) \cdot w\right)^{T} q d x$
$+\int_{0}^{L} \frac{1}{2}\left[\frac{\partial\left(N_{w}(x) \cdot w-\varphi(x) \cdot[|w|]\right.}{\partial x}-\left(N_{\theta}(x) \cdot \theta-\varphi(x) \cdot[|\theta|]\right)\right]^{T} \alpha\left[\frac{\partial\left(N_{w}(x) \cdot w-\varphi(x) \cdot[|w|]\right.}{\partial x}-\left(N_{\theta}(x) \cdot \theta-\varphi(x) \cdot[|\theta|]\right)\right] d x$
$+\int_{\Gamma} M_{S}([|\theta|]) d \Gamma+\int_{\Gamma} V_{S}[|w|]([|w|]) d \Gamma$

El término $\alpha=A_{s} G_{s}$, es la rigidez a cortante, en la que $A_{s}$ es el área efectiva a cortante y $G_{s}$ es el módulo de rigidez a cortante. Las funciones $N$ y $\varphi(x)$, así como las matrices $B$ y $B_{C}$ se definen respectivamente como:

$$
\begin{aligned}
& N_{w}=N_{\theta}=\left[\begin{array}{ll}
N_{1} & N_{2}
\end{array}\right], B_{\theta}=B_{w}=B=\frac{\partial N}{\partial x}, \quad N_{1}=\frac{L-x}{L}, N_{2}=\frac{x}{L} \\
& \varphi(x)=\frac{x}{L}, \quad B=\frac{1}{L}\left[\begin{array}{ll}
-1 & 1
\end{array}\right], \quad B_{C}=\frac{-1}{L}
\end{aligned}
$$

Para obtener un valor extremo del funcional, la ec. (29) se deriva respecto a cada variable independiente y se iguala respectivamente a cero, obteniéndose cuatro ecuaciones que posteriormente se linealizan con series de Taylor, tal que:

$$
\begin{aligned}
& \int_{0}^{L} B^{T} \alpha B \Delta w \cdot d x-\int_{0}^{L} B^{T} \alpha B_{C}[|\Delta w|] \cdot d x-\int_{0}^{L} B^{T} \alpha N \Delta \theta \cdot d x+\int_{0}^{L} B^{T} \alpha \varphi \Delta[|\theta|] d x \\
& +\int_{0}^{L} B^{T}\left[B w-B_{C}[|w|]-N_{\theta} \theta+B_{C}[|\theta|]\right] d x-F_{e x t}=0 \\
& -\int_{0}^{L} N^{T} \alpha B \Delta w \cdot d x+\left[\int_{0}^{L} N^{T} \alpha N \cdot d x+\int_{0}^{L} B^{T} E I B \cdot d x\right] \Delta \theta+\int_{0}^{L} N^{T} \alpha B_{C} \Delta[|w|] \\
& -\left[\int_{0}^{L} N^{T} \alpha \varphi d x+\int_{0}^{L} B^{T} E I B_{C} d x\right] \Delta[|\theta|]-\int_{0}^{L} B^{T}\left(B \theta-B_{C}[|\theta|]\right)-\int_{0}^{L} N^{T}\left(B w-B_{C}[|w|]-N \theta+\varphi[|\theta|]\right) d x=0
\end{aligned}
$$


$-\int_{0}^{L} B_{C}{ }^{T} \alpha B \Delta w \cdot d x+\int_{0}^{L} B_{C}{ }^{T} \alpha B_{C} \Delta[|w|] \cdot d x+\int_{0}^{L} B_{C} \alpha N \Delta \theta-\int_{0}^{L} B_{C}{ }^{T} \alpha \varphi \Delta[|\theta|] d x$

$-\int_{0}^{L} B_{C}{ }^{T}\left(B w-B_{C}[|w|]-N \theta+\varphi[|\theta|]\right) d x+\int_{0}^{w} V s([|w|]) d[|w|]+A_{S} C_{S}^{T} \Delta[|w|]=0$

$\int_{0}^{L} \varphi^{T} \alpha B \Delta w \cdot d x-\int_{0}^{L} \varphi^{T} \alpha B_{C} \Delta[|w|]-\left[\int_{0}^{L} B_{C}{ }^{T} E I B \cdot d x+\int_{0}^{L} \varphi^{T} \alpha N \cdot d x\right] \Delta \theta$

$+\left[\int_{0}^{L} B_{C}{ }^{T} E I B_{C} d x+\int_{0}^{L} \varphi^{T} \alpha \varphi \cdot d x\right] \Delta[|\theta|]-\int_{0}^{L} B_{C}{ }^{T}\left(B \theta-B_{C}[|\theta|]\right) d x+\int_{0}^{L} \varphi^{T}\left[B w-B_{C}[|w|]-N \theta+\varphi\right][|\theta|] d x$

$\int_{0}^{[|\theta|]} M(\theta) d[|\theta|]+I C_{b}^{T} \Delta[|\theta|]=0$

Sustituyendo los términos de la ec. (30) en las ecs. (31) a (34), obtiene la matriz de rigideces del elemento viga gruesa con discontinuidad interior siguiente:

$\left[\begin{array}{cccccc}\frac{\alpha}{L} & -\frac{\alpha}{L} & \frac{\alpha}{2} & -\frac{\alpha}{2} & \frac{\alpha}{L} & -\frac{\alpha}{2} \\ -\frac{\alpha}{L} & \frac{\alpha}{L} & \frac{\alpha}{2} & -\frac{\alpha}{2} & -\frac{\alpha}{L} & \frac{\alpha}{2} \\ \frac{\alpha}{2} & \frac{\alpha}{2} & \frac{E I}{L}+\frac{\alpha L}{3} & -\frac{E I}{L}+\frac{\alpha L}{6} & \frac{\alpha}{2} & \frac{E I}{L}-\frac{\alpha L}{6} \\ -\frac{\alpha}{2} & -\frac{\alpha}{2} & -\frac{E I}{L}+\frac{\alpha L}{6} & \frac{E I}{L}+\frac{\alpha L}{3} & \frac{\alpha}{2} & \frac{E I}{L}-\frac{\alpha L}{3} \\ \frac{\alpha}{L} & -\frac{\alpha}{L} & \frac{\alpha}{2} & \frac{\alpha}{2} & \frac{\alpha}{L}+A_{S} C_{S}^{T} & -\frac{\alpha}{2} \\ -\frac{\alpha}{2} & \frac{\alpha}{2} & \frac{E I}{L}-\frac{\alpha L}{6} & \frac{E I}{L}-\frac{\alpha L}{3} & -\frac{\alpha}{2} & \frac{E I}{L}+\frac{\alpha L}{3}+I C_{b}^{T}\end{array}\right]^{(n-1)}\left\{\begin{array}{c}\Delta w_{1} \\ \Delta w_{2} \\ \Delta \theta_{1} \\ \Delta \theta_{2} \\ {[|w|]} \\ {[|\theta|]}\end{array}\right\}=\left\{\begin{array}{c}f_{1} \\ f_{2} \\ m_{1} \\ m_{2} \\ f_{S} \\ m_{S}\end{array}\right\}$

donde

$\left\{\begin{array}{l}f_{1} \\ f_{2}\end{array}\right\}^{(n-1)}=\left[\begin{array}{l}F_{\text {ext1 }} \\ F_{\text {ext2 }}\end{array}\right]^{(n)}-\left[\begin{array}{cc}\frac{\alpha}{L} & -\frac{\alpha}{L} \\ -\frac{\alpha}{L} & \frac{\alpha}{L}\end{array}\right]\left\{\begin{array}{l}w_{1} \\ w_{2}\end{array}\right\}^{(n-1)}+\left[\begin{array}{c}-\frac{\alpha}{2} \\ \frac{\alpha}{2}\end{array}\right][|w|]^{n-1}+\left[\begin{array}{cc}\frac{\alpha}{2} & -\frac{\alpha}{2} \\ -\frac{\alpha}{2} & \frac{\alpha}{2}\end{array}\right]\left\{\begin{array}{l}\theta_{1} \\ \theta_{2}\end{array}\right\}^{(n-1)}-\left[\begin{array}{c}-\frac{\alpha}{2} \\ \frac{\alpha}{2}\end{array}\right][|\theta|]^{n-1}$

$\left\{\begin{array}{l}m_{1} \\ m_{2}\end{array}\right\}=0+\left[\begin{array}{cc}-\frac{\alpha}{L} & \frac{\alpha}{L} \\ -\frac{\alpha}{L} & \frac{\alpha}{L}\end{array}\right]\left\{\begin{array}{l}w_{1} \\ w_{2}\end{array}\right\}^{(n-1)}-\left[\begin{array}{ll}\left(\frac{\alpha L}{3}+\frac{E I}{L}\right) & \left(\frac{\alpha L}{6}-\frac{E I}{L}\right) \\ \left(\frac{\alpha L}{6}-\frac{E I}{L}\right) & \left(\frac{\alpha L}{3}+\frac{E I}{L}\right)\end{array}\right]\left\{\begin{array}{l}\theta_{1} \\ \theta_{2}\end{array}\right\}^{(n-1)}-\left[\begin{array}{c}-\frac{\alpha}{2} \\ \frac{\alpha}{2}\end{array}\right][|w|]^{n-1}+\left[\begin{array}{c}\frac{\alpha L}{6} \\ \frac{\alpha L}{3}\end{array}\right][|\theta|]^{n-1}$

$f_{S}^{(n-1)}=0+\left[-\frac{\alpha}{L} \frac{\alpha}{L}\right]\left\{\begin{array}{l}w_{1} \\ w_{2}\end{array}\right\}^{(n-1)}-\left[\begin{array}{ll}\frac{\alpha}{2} & \frac{\alpha}{2}\end{array}\right]\left\{\begin{array}{l}\theta_{1} \\ \theta_{2}\end{array}\right\}^{(n-1)}-\left[\frac{\alpha}{L} \mid[|w|]^{n-1}+\left[\frac{\alpha}{2}\right][|\theta|]^{n-1}-V([|w|])^{n-1}\right.$

$m_{S}^{(n-1)}=0+\left[\frac{\alpha}{2}-\frac{\alpha}{2}\right]\left\{\begin{array}{l}w_{1} \\ w_{2}\end{array}\right\}^{(n-1)}+\left[\left(\frac{\alpha L}{6}-\frac{E I}{L}\right)\left(\frac{\alpha L}{3}+\frac{E I}{L}\right)\right]\left\{\begin{array}{l}\theta_{1} \\ \theta_{2}\end{array}\right\}^{(n-1)}+\left[\frac{\alpha}{2}\right][|w|]^{n-1}-\left[\frac{\alpha L}{3}+\frac{E I}{L}\right][|\theta|]^{n-1}$

$-M([|\theta|])^{n-1}$

donde $C_{S}^{T}=\partial V([|w|]) / \partial[|w|]$ y $C_{b}^{T}=\partial M([|\theta|]) / \partial[|\theta|]$ son respectivamente los operadores tangente en el salto del desplazamiento transversal y rotacional. 


\section{Agrupamiento de los elementos barra y viga con discontinuidades interiores}

Los elementos viga-columna con discontinuidades interiores se obtienen agrupando los grados de libertad (GDL) del elemento barra con el de las vigas. En el caso del elemento viga-columna delgada tienen ocho GDL, como se muestra en la Figura 6a, en la que los GDL 7 y 8 corresponden respectivamente a los saltos $[|\mathrm{u}|]$ y $[|\theta|]$. Por otra parte, la viga-columna gruesa tienen nueve GDL, como se muestra en la Figura $6 \mathrm{~b}$, en la que los GDL 7, 8 y 9 corresponden respectivamente a los saltos [|u|], [|w|] y $[|\theta|]$.

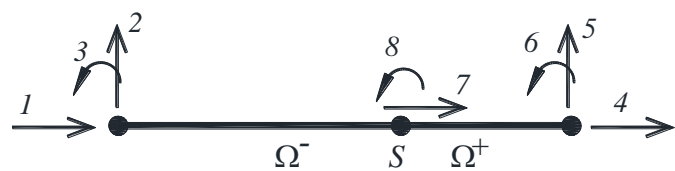

a)

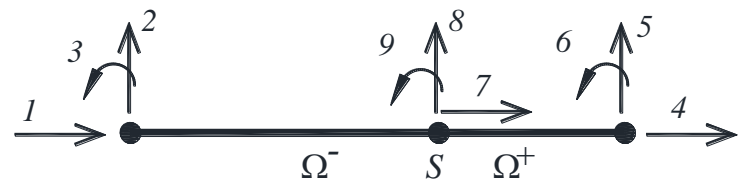

b)

Figura 6. GDL en elementos viga-columna con discontinuidad: a) delgadas y b) gruesas

Los términos de la matriz del elemento finito barra de la ec. (10) se agrupan respectivamente con los elementos viga delgada de la ec. (23) y con los de la viga gruesa de la ec. (35), tal que se tienen los elementos viga-columna con discontinuidad interior delgada y gruesa, respectivamente:

\begin{tabular}{|c|c|c|c|c|c|c|c|c|c|c|}
\hline$\frac{A E}{L}$ & 0 & 0 & $-\frac{A E}{L}$ & 0 & 0 & $\frac{A E}{L}$ & 0 & & & \\
\hline 0 & $\frac{12 E I}{L^{3}}$ & $\frac{6 E I}{L^{2}}$ & 0 & $-\frac{12 E I}{L^{3}}$ & $\frac{6 E I}{L^{2}}$ & 0 & 0 & & & \\
\hline 0 & $\frac{6 E I}{L^{2}}$ & $\frac{4 E I}{L}$ & 0 & $-\frac{6 E I}{L^{2}}$ & $\frac{2 E I}{L}$ & 0 & $\frac{E I}{L}$ & $\begin{array}{l}\Delta d_{1} \\
\Delta w_{1}\end{array}$ & & $f_{1}$ \\
\hline$-\frac{A E}{L}$ & 0 & 0 & $\frac{A E}{L}$ & 0 & 0 & $-\frac{A E}{L}$ & 0 & $\begin{array}{l}\Delta \theta_{1} \\
\Delta d_{2}\end{array}$ & & $\begin{array}{l}m_{1} \\
n_{2}\end{array}$ \\
\hline 0 & $-\frac{12 E I}{L^{3}}$ & $\frac{6 E I}{L^{2}}$ & 0 & $\frac{12 E I}{L^{3}}$ & $-\frac{6 E I}{L^{2}}$ & 0 & 0 & $\begin{array}{l}\Delta w_{2} \\
\Delta \theta_{2}\end{array}$ & & $\begin{array}{l}f_{2} \\
m_{2}\end{array}$ \\
\hline 0 & $\frac{6 E I}{L^{2}}$ & $\frac{2 E I}{L}$ & 0 & $-\frac{6 E I}{L^{2}}$ & $\frac{4 E I}{L}$ & 0 & $-\frac{E I}{L}$ & $\begin{array}{l}\Delta|u| \\
\Delta \theta\end{array}$ & $\square$ & $\begin{array}{l}n_{S} \\
m_{s}\end{array}$ \\
\hline$\frac{A E}{L}$ & 0 & 0 & $-\frac{A E}{L}$ & 0 & 0 & $\frac{A E}{L}+A C_{N}^{T}$ & 0 & & & \\
\hline 0 & 0 & $\frac{E I}{L}$ & 0 & 0 & $-\frac{E I}{L}$ & 0 & $\frac{E I}{L}+I C_{b}^{T}$ & & & \\
\hline
\end{tabular}




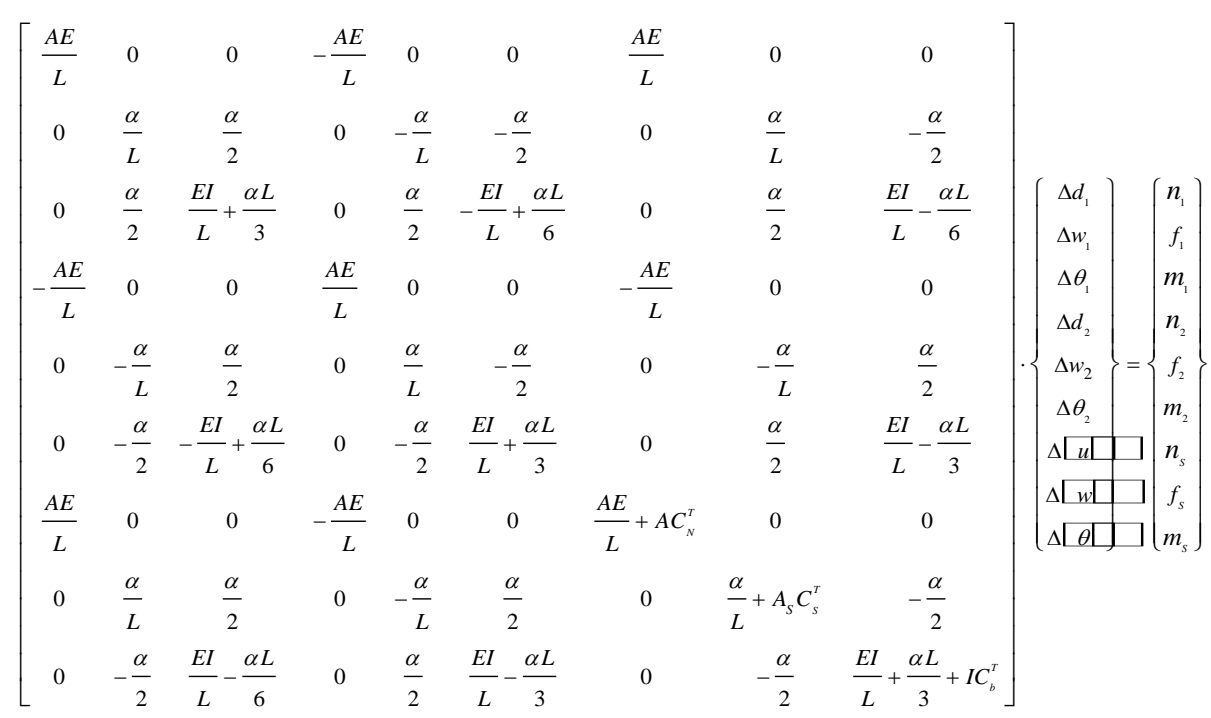

Es importante mencionar que las acciones axiales son independientes de las rotaciones o desplazamientos transversales en las ecs. (37) y (38).

\section{Condensación estática}

Las ecs. (37) y (38) se pueden expresar como:

$\left[\begin{array}{cc}K_{e e} & K_{e[e \mid]]} \\ K_{[|e|] e} & K_{[|e|][e]]}\end{array}\right]\left\{\begin{array}{c}\Delta d_{e} \\ \Delta[|e|]\end{array}\right\}=\left\{\begin{array}{c}F_{n} \\ R_{[|e|]}\end{array}\right\}$

donde $K_{e e}, K_{e[\mid e]}, K_{[\mid e]] e}$ y $K_{[\mid e][\mid[e]]}$ son las submatrices asociadas a los GDL, incluyendo la discontinuidad del elemento. $F_{n}$ y $R_{[\mid e]]}$ son los vectores de fuerzas y momentos nodales, respectivamente. $\Delta d_{e}$ son los desplazamientos en los nodos y $\Delta[|e|]$ los saltos en la discontinuidad.

La ec. (39) se puede reescribir de acuerdo con Tena-Colunga (2007), como:

$$
\begin{aligned}
& F_{n}=K_{e e} \Delta d_{e}+K_{e[e \mid]]} \Delta[|e|] \\
& R_{[[e \mid]}=K_{[l e]]} \Delta d_{e}+K_{[|e|][|e|]} \Delta[|e|]
\end{aligned}
$$

Despejando $\Delta[|e|]$ de la ec. (41) se obtiene:

$$
\Delta[|e|]=K_{[|e|][e \mid e]}^{-1} R_{[[e \mid]}-K_{[[e][|e|]]}^{-1} K_{[[e]]} \Delta d_{e}
$$

sustituyendo la ec. (42) en la ec. (40) se tiene:

$$
\left[K_{e e}-K_{e[|e|]} K_{[|e|][|e|]}^{-1} K_{[|e|] e}\right]\left[\Delta d_{e}\right]=\left[F_{n}-K_{e[|e|]} K_{[|e|][e \mid]]}^{-1} R_{[l e]]}\right]
$$

que se representa como: 


$$
\left[K_{\Delta}\right]\{\Delta d\}=\left[R^{*}\right]
$$

la matriz condensada de rigideces, $K_{\Delta}$, se define de la ec. (43) como:

$$
\left[K_{\Delta}\right]=\left[K_{e e}-K_{e e} K_{e e}^{-1} K_{e e}\right]
$$

y el vector condensado de las fuerzas residuales $R^{*}$ es:

$$
\left[R^{*}\right]=\left[F_{n}-K_{e_{e}} K_{e}^{-1} R_{e}\right]
$$

Las matrices de los elementos viga-columna con discontinuidades interiores delgada y gruesa definidas respectivamente en las ecs. (37) y (38) se pueden condensar estáticamente como la ec. (43), de tal manera que sólo queden activos los GDL de los extremos del elemento, y los GDL de los saltos queden condensados con base en las ecs. (44) a (46).

\section{MODELOS CONSTITUTIVOS}

Los modelos constitutivos de daño se utilizan para representar el comportamiento idealizado de los materiales en sus intervalos elástico y no lineal, después de alcanzar una superficie de falla, en el que las propiedades mecánicas del material se degradan. El modelo de daño se clasifica como continuo cuando la degradación del material se distribuye en todo el volumen del elemento finito. Por el contrario, en los modelos de daño discreto la degradación del material se localiza en una zona de espesor cero dentro de los elementos. Los modelos constitutivos de daño continuos se formulan respecto a las fuerzas o los esfuerzos contra los desplazamientos o las deformaciones, pero los modelos de daño discreto se formulan respecto a las fuerzas o los esfuerzos contra los saltos, como se muestran respectivamente en la Figura 7.

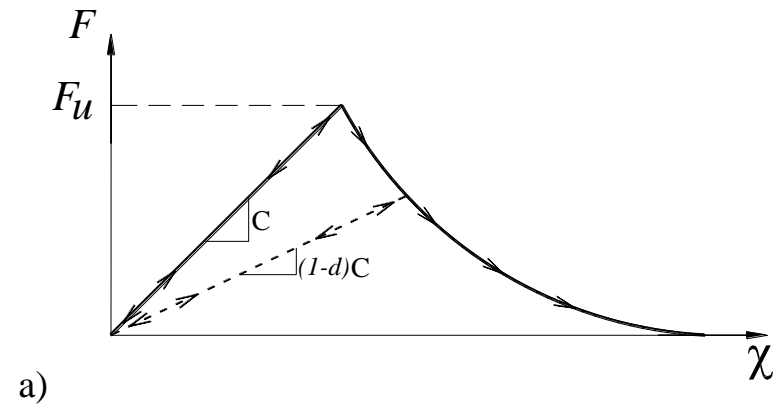

Figura 7. Modelo de daño: a) continuo b) discreto

El modelo de daño isótropo para elementos viga formulado por Juárez y Ayala (2012) se define como: 


$$
\begin{array}{cc}
\begin{array}{c}
\text { Densidad de energía libre } \\
\text { Ecuación constitutiva }
\end{array} & F=\frac{\partial \psi(\chi, r)}{\partial \chi}=(1-d) C: \chi \\
\text { Variable de daño } & d(r)=1-\frac{q}{r} ; \quad d \in[0,1] ; \quad q \in\left[0, r_{O}\right] \\
\text { Evolución de daño } & \dot{r}_{(t)}=\gamma\left\{\begin{array}{c}
r \in\left[r_{O}, \infty\right] \\
r_{O}=\left.r\right|_{t=0}=\frac{F_{u}}{\sqrt{C}}
\end{array}\right. \\
\text { Criterio de daño } & f\left(\tau_{F}, q\right)=\tau_{F}-q=\sqrt{F: C^{-1}: F}-q ; \quad\left\{\begin{array}{l}
\left.q \in r_{0}\right] \\
\left.q\right|_{t=0}=r_{0}
\end{array}\right. \\
\text { Regla de ablandamiento } & \dot{q}=H^{d}(r) \dot{r} ; \quad H^{d}(r)=q^{\prime}(r) \leq 0
\end{array}
$$

donde $\Psi$ es la densidad de energía libre, representada por el área bajo la curva del modelo constitutivo, $\chi$ representa el vector de desplazamientos, $C$ son las constantes elásticas, $F$ el vector de fuerzas, $d$ es la variable de daño que depende de la variable de ablandamiento $q, H$ es el parámetro de ablandamiento, $\gamma$ es el multiplicador de daño que determina la condición de carga o descarga y $r$ es la variable de estado. La función $f\left(\tau_{F}, q\right)$ limita la superficie de falla del material, en el espacio de fuerzas.

El modelo de daño discreto para elementos viga es:

$$
\begin{aligned}
& \text { Energía libre discreta } \quad \psi([|u|], \bar{\alpha})=(1-\omega) \psi_{0}([|u|]), \quad \psi_{0}([|u|])=\int_{0}^{[|u|]} T([|u|]) d[|u|] \\
& \text { Ecuación constitutiva } \quad F=\frac{\partial \psi([|u|], \bar{\alpha})}{\partial[|u|]}=(1-\omega) C[|u|] \\
& \text { Variable de daño } \\
& \omega=1-\frac{\bar{q}(\bar{\alpha})}{\bar{\alpha}} ; \omega \in[-\infty, 1] \\
& \text { Ley de evolución del daño } \\
& \begin{array}{c}
\dot{\bar{\alpha}}=\bar{\lambda}=\frac{\partial}{\partial t}(\bar{\alpha}) ; \quad \bar{\alpha} \in(0, \infty) \\
f(T, \bar{q})=\tau_{T}-\bar{q} ; \quad \tau_{T}=\|F\|(C)^{-1}=\sqrt{F[C]^{-1} F} \\
\dot{\bar{q}}(\bar{\alpha})=\bar{H} \dot{\bar{\alpha}} ; \quad \bar{H}=\bar{q}(\bar{\alpha}) \leq 0 \\
f \leq 0 ; \quad \bar{\lambda} \geq 0 ; \quad \bar{\lambda} f=0 \\
\bar{\lambda} \dot{f}=0
\end{array} \\
& \text { Criterio de daño } \\
& \text { Regla de ablandamiento } \\
& \text { Condición de carga-descarga } \\
& \text { Consistencia }
\end{aligned}
$$

donde $\omega$ es la variable de daño definida en términos de la variable de ablandamiento. El resto de las variables se definen de igual forma que las del modelo de daño continuo pero con el término discreto, e.g., $\bar{H}$ es el parámetro de ablandamiento discreto.

La razón de cambio de fuerzas entre los desplazamientos se define como un operador constitutivo tangente de un modelo continuo, $C^{T}$, y la razón de cambio de fuerzas entre los saltos de desplazamientos corresponde al operador constitutivo discreto del modelo discreto $C_{d}^{T}$, como se definen, respectivamente, en las ecuaciones siguientes:

$$
C^{T}=\frac{\partial F}{\partial \chi}, \quad C_{d}^{T}=\frac{\partial F}{\partial[|\chi|]}
$$


Cuando un elemento se encuentra en la condición de carga, en intervalo no lineal, los operadores constitutivos tangentes se calculan con la ec. (50), y para el intervalo elástico o condición de descarga, con la ec. (51).

$$
\begin{aligned}
& C^{T}=(1-d) C-\frac{q-H r}{r^{3}}(C: \chi \otimes \chi: C), \quad C_{d}^{T}=(1-\omega) C-\frac{\bar{q}-H \bar{\alpha}}{\bar{\alpha}^{3}}(C \cdot[|u|] \otimes[|u|] \cdot C) \\
& C^{T}=(1-d) C, \quad C_{d}^{T}=(1-\omega) C
\end{aligned}
$$

Los elementos viga-columna requieren de modelos constitutivos continuos con variables de la fuerza normal, $N$, contra la deformación, $\varepsilon$, o modelos discretos, $N$-[ $[u \mid]$, como se muestra en la figura 8; además se requiere de modelos constitutivos continuos con variables de la fuerza cortante, $V$, contra la deformación por cortante, $\gamma$, o modelos discretos, $V$-[|w|], como se muestra en la Figura 9; y se requiere de modelos constitutivos continuos con variables del momento, $M$, contra la curvatura, $\kappa$, o modelos discretos, $M$-[ $|\theta|]$, como se muestra en la Figura 10.

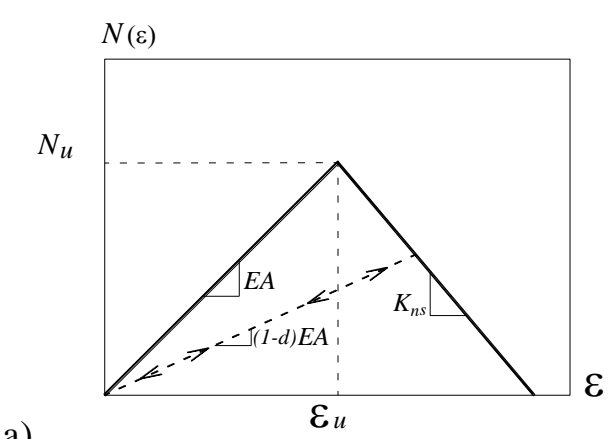

a)

Figura 8. Comportamiento constitutivo fuerza normal contra: a) deformación axial y b) salto desplazamiento axial

a)

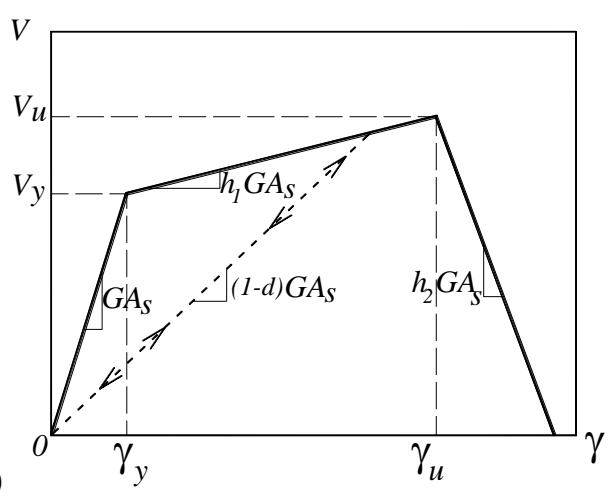

b)

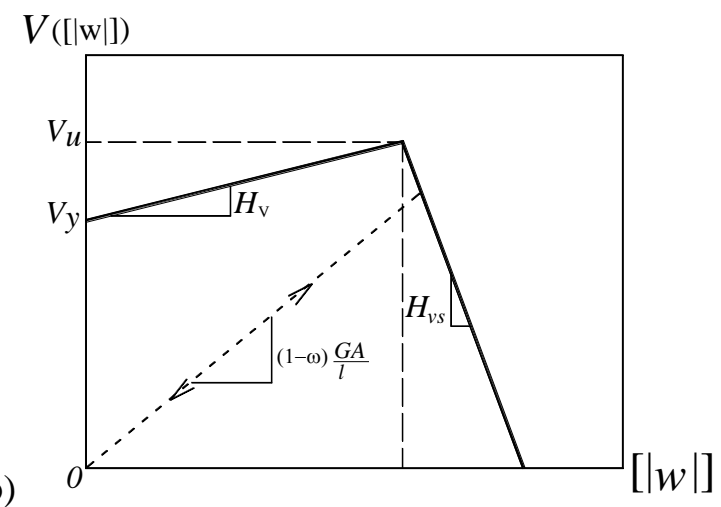

Figura 9. Comportamiento constitutivo cortante contra: a) deformación por cortante y b) salto desplazamiento transversal 
a)

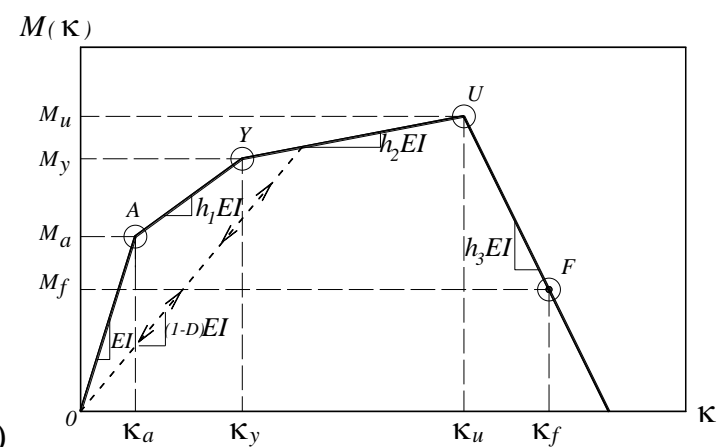

b)

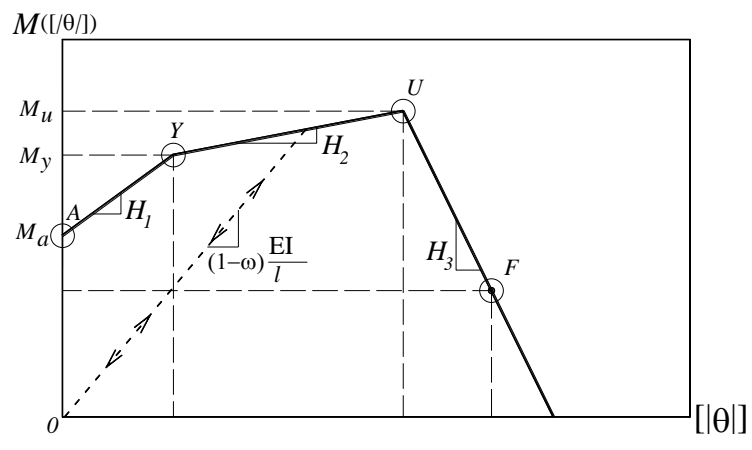

Figura 10. Comportamiento constitutivo momento contra: a) curvatura y b) salto rotación

Los elementos viga-columna con discontinuidades interiores desarrollados en la sección 0 , así como los modelos constitutivos descritos en esta sección, se implantaron en FEAP, acrónimo de su nombre en el idioma inglés, Finite Element Analysis Program, el cual fue desarrollado por Taylor (2008).

\section{EJEMPLOS NUMÉRICOS}

\section{Viga delgada en voladizo}

\section{Sujeta a momento}

En este ejemplo, la viga en voladizo que se muestra en la Figura 11a se somete a un momento, $M$, incremental en el extremo libre. La viga tiene una sección transversal simplemente reforzada como se muestra en la Figura 12a, que en la parte inferior tiene 4 barras longitudinales de acero del número 4. Las propiedades mecánicas del concreto son: módulo de Young $E_{c}=37.272 \mathrm{GPa}$ y esfuerzo a compresión del concreto $f^{\prime}{ }^{\prime}=38 \mathrm{MPa}$. El acero de refuerzo tiene las propiedades mecánicas siguientes: módulo de Young $E_{s}=200 \mathrm{GPa}$ y esfuerzo de fluencia $f_{y}=400 \mathrm{MPa}$.

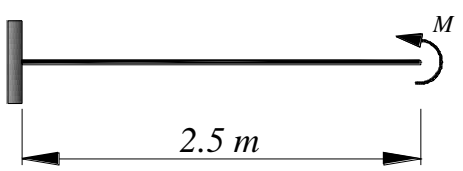

a)

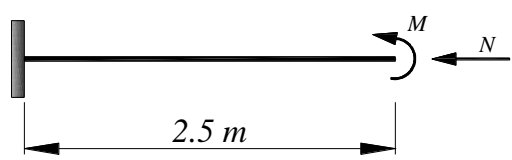

b)

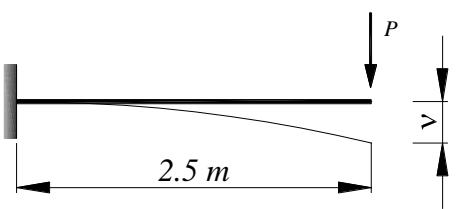

c)

Figura 11. Geometría y aplicación de fuerzas (adaptada de Jukic et al. 2013)

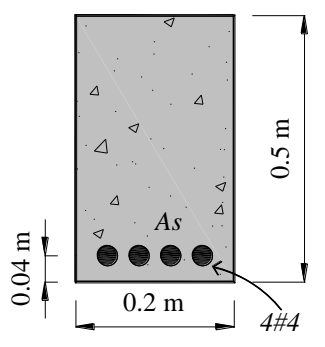

a)

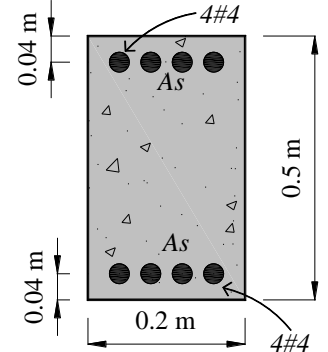

b)

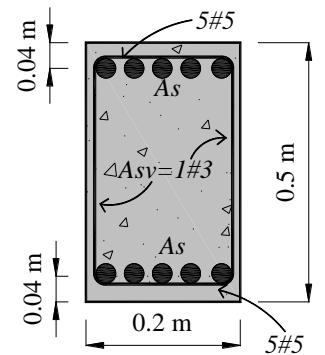

c)

Figura 12. Secciones transversales de vigas: a) simplemente reforzada, b) doblemente reforzada con barras del número 4 y c) doblemente reforzada con barras del número 5 
La viga se discretizó con diez elementos viga-columna delgada y once nodos, como se muestra en la Figura 13, por lo que cada elemento tiene una longitud de veinticinco centímetros. La sección transversal de la viga, mostrada en la Figura 12a, tiene un comportamiento constitutivo continuo del $M$ contra la $\kappa$, como se muestra en la Figura 14a, y un comportamiento discreto del $M$ contra el [ $|\theta|]$, como se muestra en la Figura 14b.

(1) (2) (3) (4) (5) (6) (7) (8) (9)

Figura 13. Discretización con elementos finitos de viga

a)

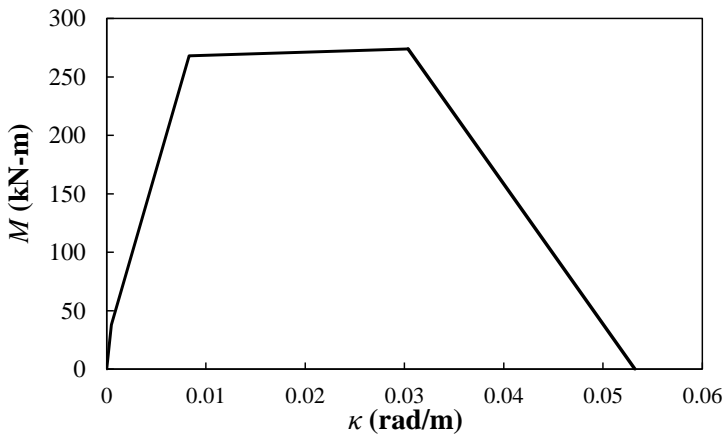

b)

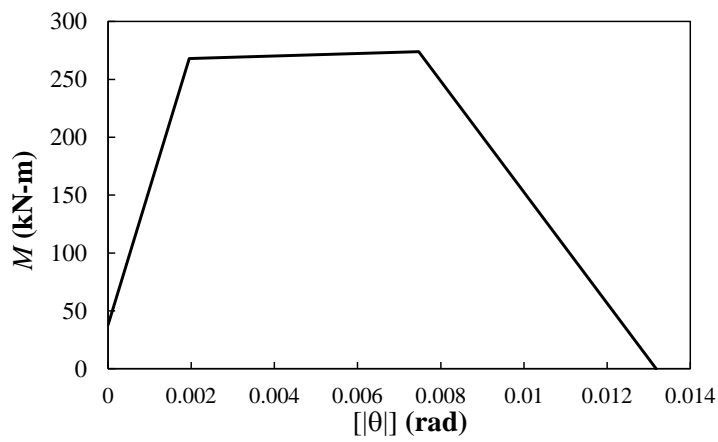

Figura 14. Comportamientos constitutivos: a) $M-\kappa$ y b) $M-\theta$

Para la solución de este problema, se aplicó gradualmente la rotación, $\theta$, en el extremo derecho de la viga, donde $M$ se calculó como la reacción en ese apoyo. Debido a que $M$ es constante a lo largo de la viga, todos los elementos desarrollan una articulación simultáneamente como se muestra en la Figura 15, por lo que la curva $M$ contra [ $|\theta|]$ es idéntica en todos los elementos con los que se discretizó el modelo numérico, como la que se muestra en la Figura 14b. La curva del $M$ contra la $\theta$, impuesta en el extremo derecho, se muestra en la Figura 16, la cual es congruente con la obtenida por Jukic (2013), quién realizó el mismo ejemplo utilizando elementos vigas de Euler-Bernoulli con discontinuidades. El área bajo la curva $M$-[ $[\theta \mid]$ en la Figura $14 \mathrm{~b}$ es de $2.58 \mathrm{kN}-\mathrm{M}$, la cual corresponde a la energía disipada durante la formación completa de una articulación, por lo que en la formación de las diez articulaciones se disipa una energía de $25.8 \mathrm{kN}$ M. Por otra parte, el trabajo externo que se proporciona a la viga corresponde al área bajo la curva $M-\theta$ de la Figura 16, que aproximadamente es de $25.8 \mathrm{kN}-\mathrm{M}$, lo que garantiza que el trabajo inducido a una estructura se disipa adecuadamente como la energía necesaria para generar discontinuidades, articulaciones, dentro de ella.

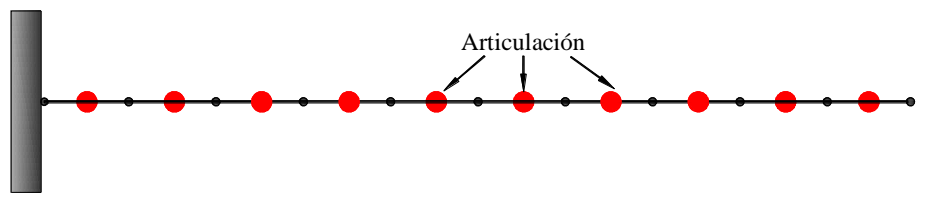

Figura 15. Formación de articulaciones 


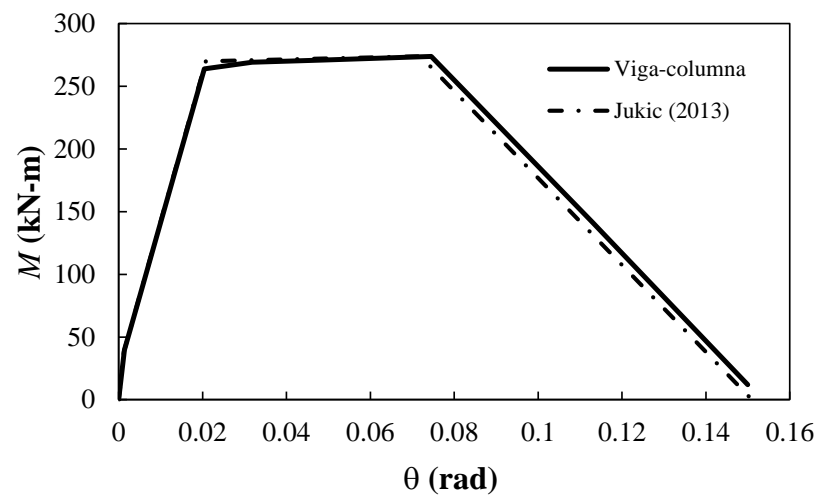

Figura 16. Curva de $M-\theta$ en el extremo derecho

\section{Sujeta a momento y fuerza axial}

En este ejemplo, a la viga en voladizo mostrada en la Figura $11 \mathrm{~b}$ se le aplicó primeramente una carga axial en compresión de $\mathrm{N}_{c}=100 \mathrm{kN}$ y, posteriormente, se impuso gradualmente $M$ en el extremo de la viga como se muestra en la Figura 11b. Al igual que el ejemplo anterior, el modelo se discretizó con 10 elementos finitos, como se muestra en la Figura 15, y la sección transversal de la viga es la que se muestra en la Figura 12a. Sin embargo, la fuerza normal modifica el comportamiento constitutivo continuo del $M$ contra la $\kappa$, pues incrementa la capacidad de momento de la sección y se reduce la capacidad de la rotación, como se

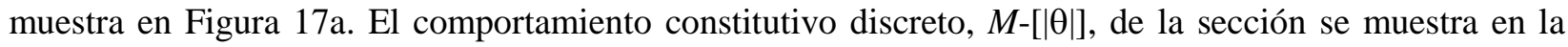
Figura 17b. Por otra parte, el comportamiento constitutivo continuo, $N-\varepsilon$, se muestra en la Figura 18a y el discreto, N-[|u|], se muestra en la Figura 18b.

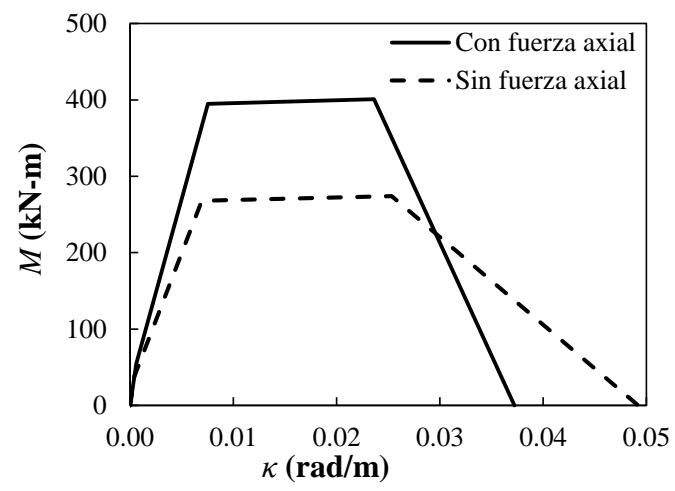

b)

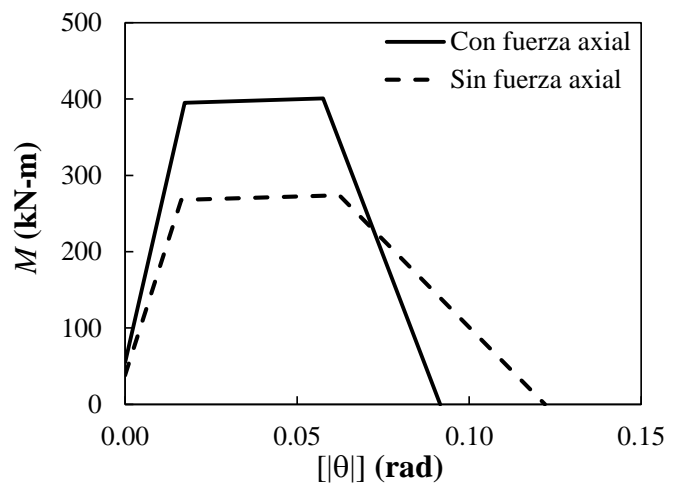

Figura 17. Comportamientos constitutivos: a) $M-\kappa$ y b) $M-\theta$ 
a)

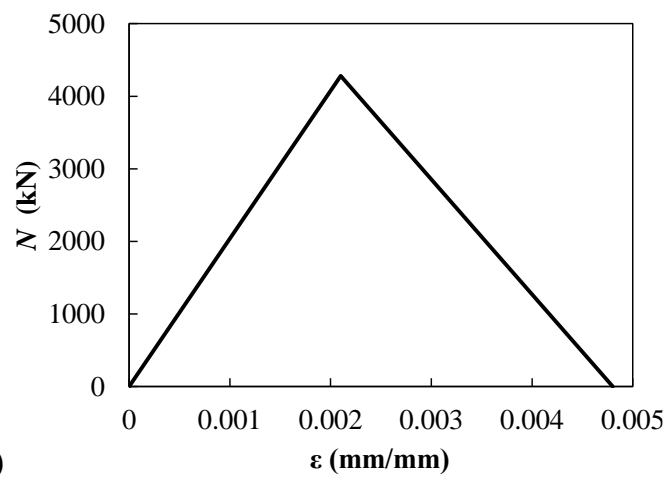

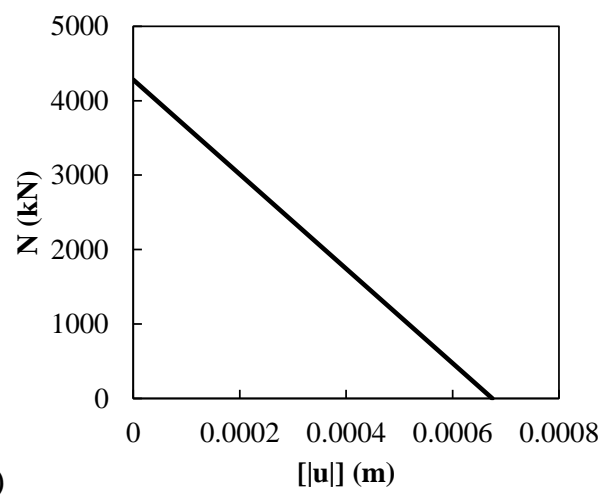

b)

Figura 18. Comportamientos constitutivos: a) $N-\varepsilon$ y b) $N-[|u|]$

Al aplicar gradualmente la $\theta$ en el extremo derecho de la viga, se obtiene la curva de $M-\theta$, que se compara con la curva obtenida por Jukic (2013), como se muestran en la Figura 19, donde se observa que ambas curvas son congruentes hasta una rotación de $0.57 \mathrm{rad}$; sin embargo, para rotaciones mayores, se presentan diferencias que se atribuyen a los modelos constitutivos distintos utilizados en las respectivas

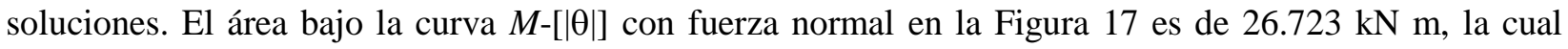
corresponde a la energía disipada durante la formación completa de una articulación. Por otra parte, el trabajo inducido al sistema es de $26.723 \mathrm{kN} \mathrm{m}$, que corresponde al área bajo la curva $\mathrm{M}-\theta$ con fuerza normal en la Figura 19, por lo que la viga disipa adecuadamente la energía mediante la formación de articulaciones.

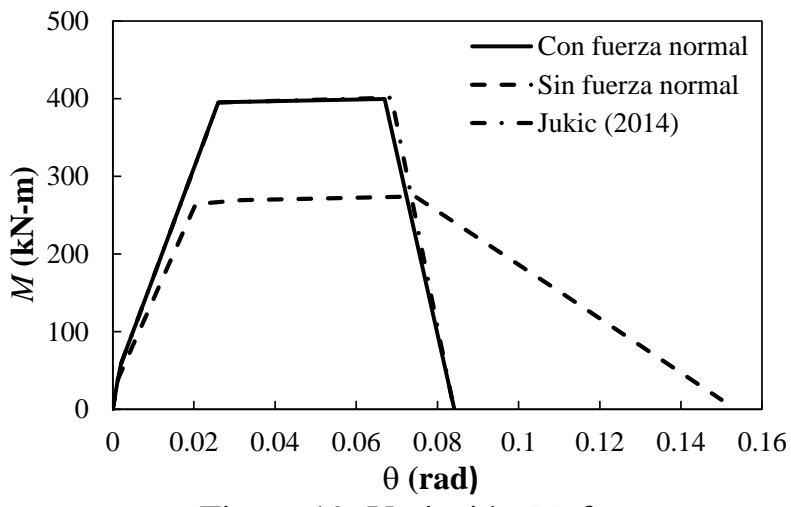

Figura 19. Variación $M-\theta$

\section{Sometida a carga vertical}

La viga analizada en los ejemplos anteriores, se somete a una carga vertical, $P$, en el extremo, como se muestra en la Figura 11c. Esta viga está doblemente reforzada, pues además del acero de refuerzo inferior se coloca un refuerzo en la parte superior de 4 barras de acero del número 4 , como se muestra en la Figura 12b. En este ejemplo, la viga se discretizó con uno y con diez elementos finitos. Los modelos constitutivos continuo, $M-\kappa$, y discreto, $M$-[ $[\theta \mid]$, se muestran respectivamente en la Figura 20, los cuales son distintos a los de los ejemplos anteriores debido al incremento del acero de refuerzo. Por otra parte, en la Figura 21 se muestran los respectivos comportamientos constitutivos, continuo $\mathrm{N}-\varepsilon$ y discreto $\mathrm{N}-[|\mathrm{u}|]$. 

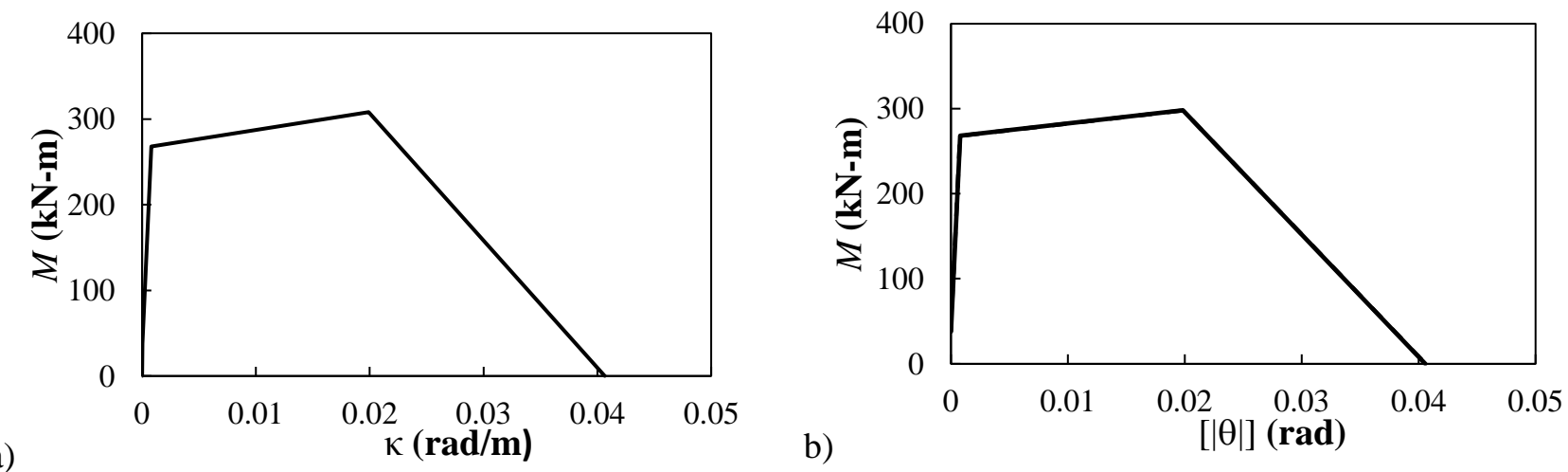

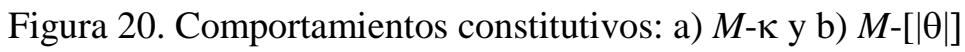
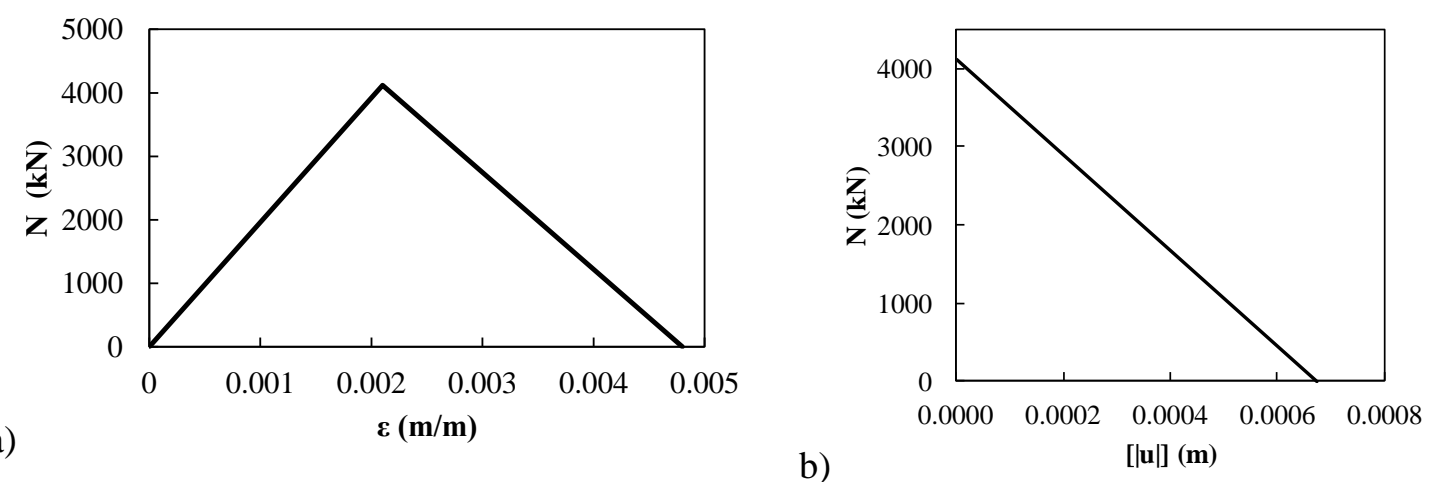

Figura 21. Comportamientos constitutivos: a) $N-\varepsilon$ y b) $N$-[|u|]

Para la solución de este problema se impuso gradualmente un desplazamiento vertical, $v$, en el extremo libre, donde la carga aplicada corresponde la reacción vertical, $P$, del apoyo. En este caso se formó una articulación en el extremo derecho de la viga, como se muestra en la Figura 22, donde la magnitud del momento es mayor. En la Figura 23 se muestra la curva de $P$, en el extremo izquierdo de la viga, contra el $v$ en el extremo libre, la cual se compara con la obtenida por Jukic (2013), donde se observa que para el elemento finito viga-columna desarrollado en este trabajo se obtiene la misma solución al discretizar la viga con uno o con diez elementos; sin embargo, la curva $P-v$ obtenida por Jukic (2013) es dependiente del número de elementos. Al integrar la curva de la $P$ contra el $v$ en la Figura 23, se tienen un trabajo externo de $8.68 \mathrm{kNm}$, que es igual a la energía que se obtiene de la curva del $M$ contra el [|$|\theta|]$ en la Figura 20b, que es la energía necesaria para desarrollar al articulación mostrada en la Figura 22.

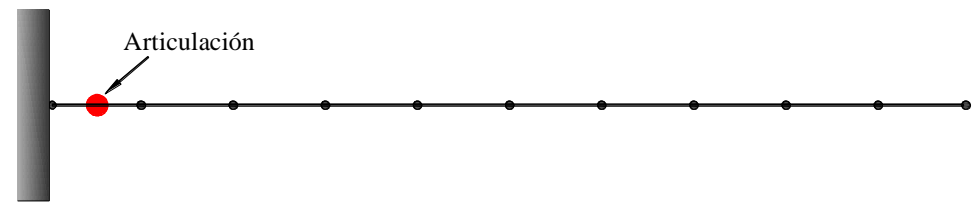

Figura 22. Formación de articulación 


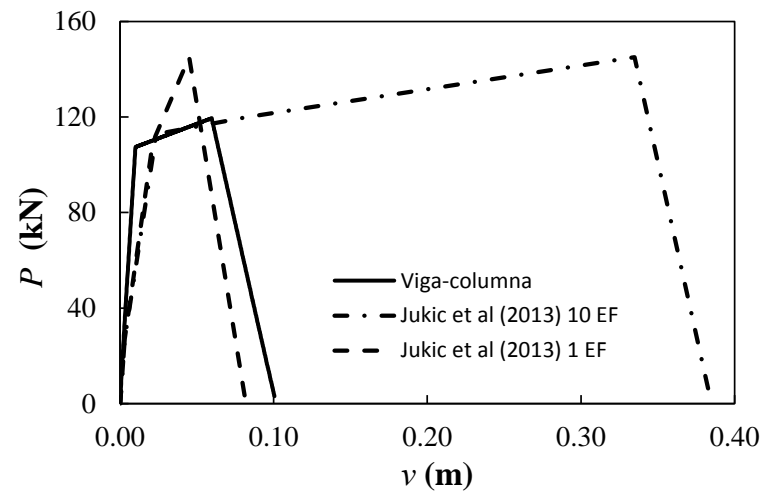

Figura 23. Curvas P-v

\section{Viga en voladizo con deformación por cortante}

En este ejemplo se analiza la misma viga en voladizo de los ejemplos anteriores, a la cual se le aplica una carga en el extremo derecho como se muestra en la Figura 11c. La sección transversal está doblemente armada con barras del número 5 y el refuerzo transversal consiste de estribos del número 3 separados a $d / 2$, donde $d$ es la altura efectiva de la sección, como se muestra en Figura 12c. Las propiedades mecánicas del concreto son: módulo de Young $E_{c}=40 \mathrm{GPa}$, esfuerzo de máximo de compresión $f_{c}^{\prime}=44.9 \mathrm{MPa}$. Las propiedades del acero de refuerzo para acero longitudinal y transversal son: módulo de Young $E_{s}=200 \mathrm{GPa}$ y esfuerzo de fluencia $f_{y}=400 \mathrm{MPa}$.

Los modelos constitutivos continuo $M$ - $\kappa$ y discreto $M$ - $[|\theta|]$ se muestran respectivamente en la Figura 24 a y b. Los modelos constitutivos continuo $V$ - $\gamma$ y discreto $V$ - $[|w|]$ se muestran respectivamente en la Figura 25 a y b. Este ejemplo se analizó utilizando las formulaciones de vigas-columnas gruesas y delgadas con discontinuidades interiores desarrolladas respectivamente en las secciones 0 y 0 . En el análisis de este ejemplo se impuso gradualmente $v$, en el extremo libre, calculando las reacciones de fuerza, $P$, en el extremo libre y el momento, $M$, en el apoyo derecho.

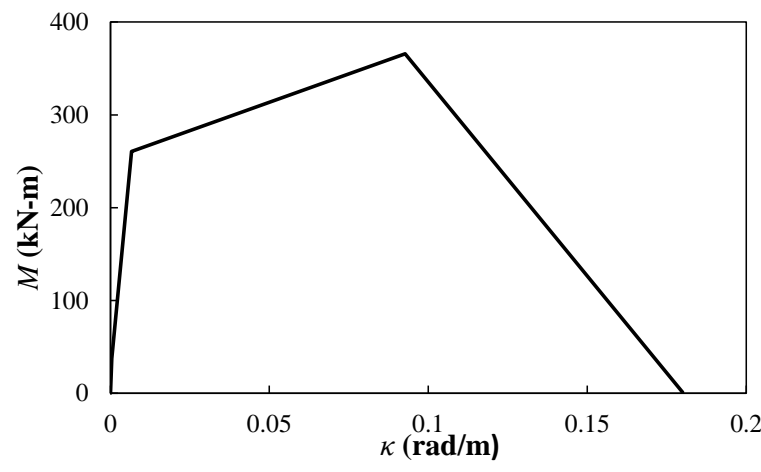

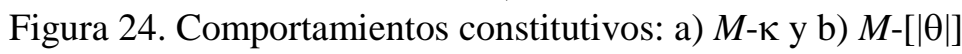

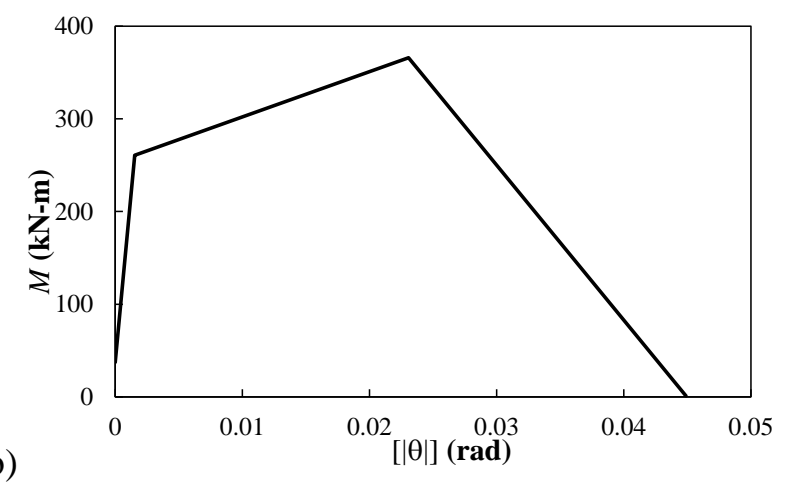

b) 

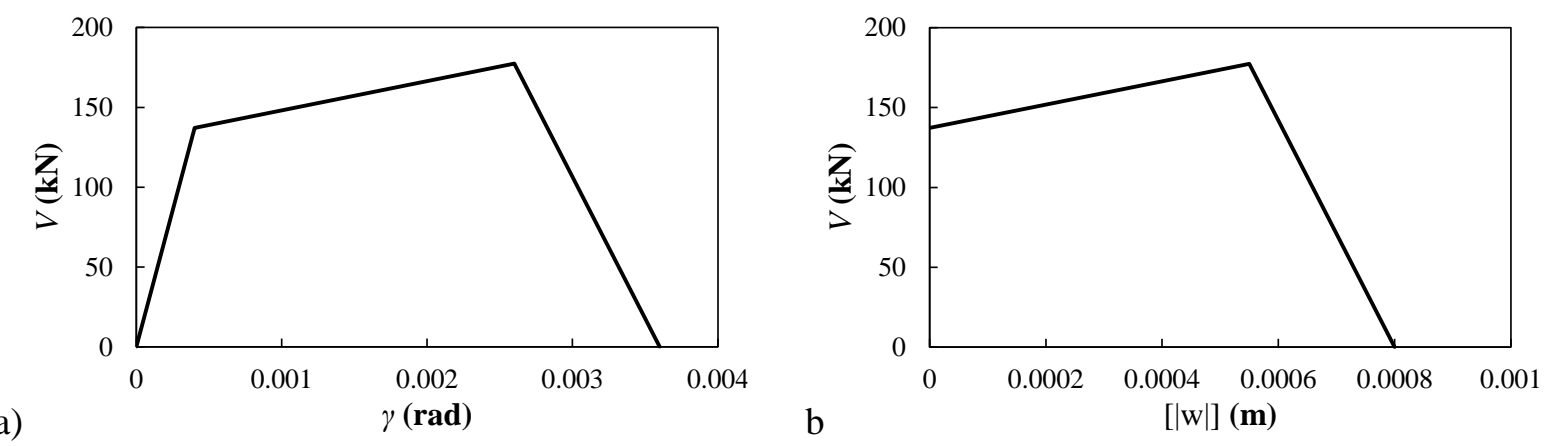

Figura 25. Comportamientos constitutivos discretos: a) $V-\gamma \mathrm{y}, \mathrm{b}) V-[|\mathrm{w}|]$

La Figura 26 muestra las curvas $P-v$ calculadas con elementos viga-columna gruesa y delgada, las cuales se comparan con la calculada por Jukic (2013), en las que se observan diferencias entre estas aproximaciones, las cuales se atribuyen a que los resultados reportados por Jukic (2013) dependen del número de elementos. Los resultados obtenidos con ambos elementos, gruesos y delgados, mantienen relación con el comportamiento constitutivo $M-\kappa$, las diferencias se atribuyen a la energía por cortante incluida en la formulación de viga-columna gruesa. Además se observa que la curva $P-v$ no alcanza la magnitud del cortante último, $V_{u}$, lo cual evidencia que no se forma la discontinuidad por cortante. El área bajo la curva $M$-[ $[\theta \mid]$ en la Figura 24 es de $10.98 \mathrm{kN} \mathrm{m}$, la cual corresponde a la energía disipada durante la formación completa de una articulación. Por otra parte, el trabajo inducido al sistema es de $8.91 \mathrm{kN} \mathrm{m}$, que corresponde al área bajo la curva $P-v$ en la Figura 26. La diferencia entre la energía necesaria para desarrollar la articulación y el trabajo inducido a la viga se atribuye a que no se genera por completo la articulación.

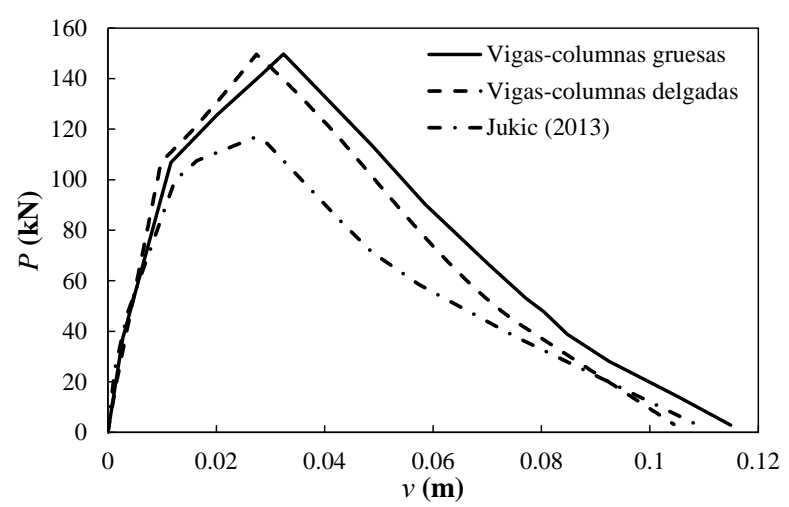

Figura 26. Curva $P-v$

\section{Marco de dos niveles}

El marco de dos niveles mostrado en la Figura 27.a, tiene una longitud $L=3.5 \mathrm{~m}$ y altura $H=2.0 \mathrm{~m}$. Las columnas del marco se someten a compresión al aplicar las cargas verticales de $700 \mathrm{kN}$ en los nodos 5 y 6, posteriormente se aplica una carga horizontal $F$ en el nodo 5. El concreto tiene las propiedades mecánicas siguientes: módulo de Young $E_{c}=28.6 \mathrm{GPa}$ y esfuerzo último a compresión $f_{c}{ }_{c}=30 \mathrm{MPa}$. Por otra parte, el acero de refuerzo tiene como propiedades mecánicas: módulo de Young $E_{s}=192.5 \mathrm{GPa}$ y esfuerzo de fluencia $f_{y}=418 \mathrm{MPa}$. Las secciones transversales y acero de refuerzo de las vigas y columnas tienen la misma área de concreto, $30 \times 40 \mathrm{~cm}$, y la misma área de acero longitudinal $A_{s}=11.36 \mathrm{~cm}^{2}$ en la parte superior e inferior, pero con recubrimientos de 4 y $3 \mathrm{~cm}$ respectivamente, como se muestran en la Figura 28. Con estas propiedades de las secciones transversales se determinaron los comportamientos 
constitutivos continuo $M-\kappa$ y discreto $M$-[ $[\theta \mid]$, que se muestran respectivamente en la Figura 29a y b. Los modelos constitutivos continuo $N-\varepsilon$ y discreto $N$-[|u|] se muestran respectivamente en la Figura 30 a y b.

a)

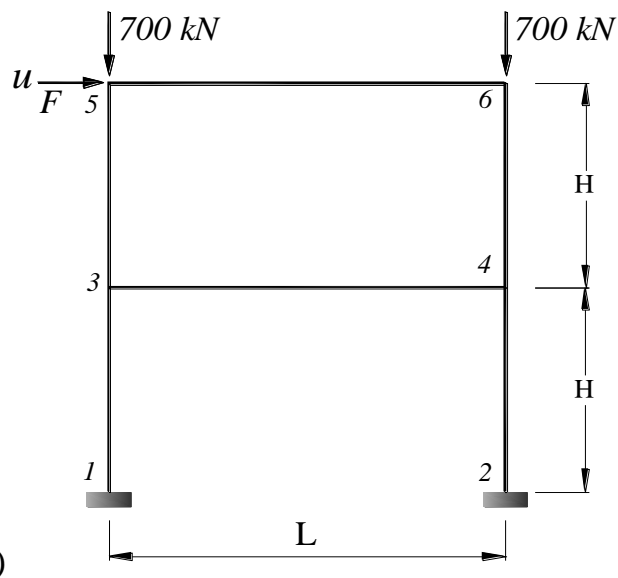

b)

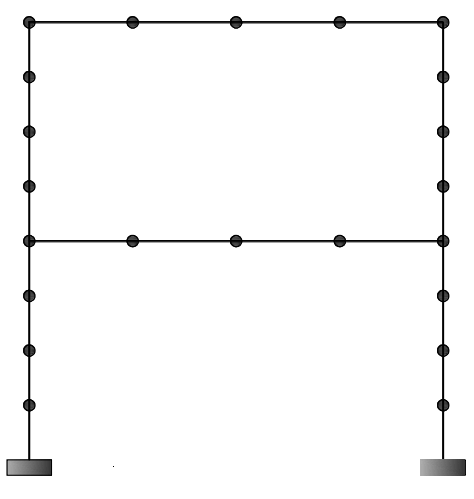

Figura 27. Marco estructural: a) geometría y b) discretización

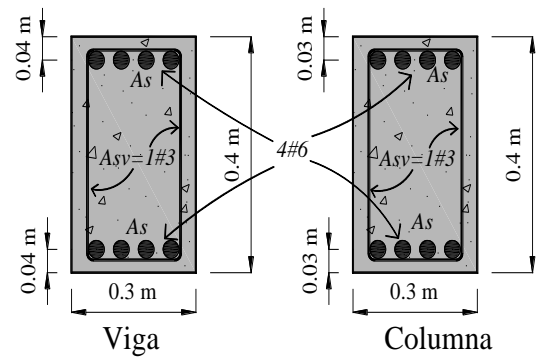

Figura 28. Secciones transversales
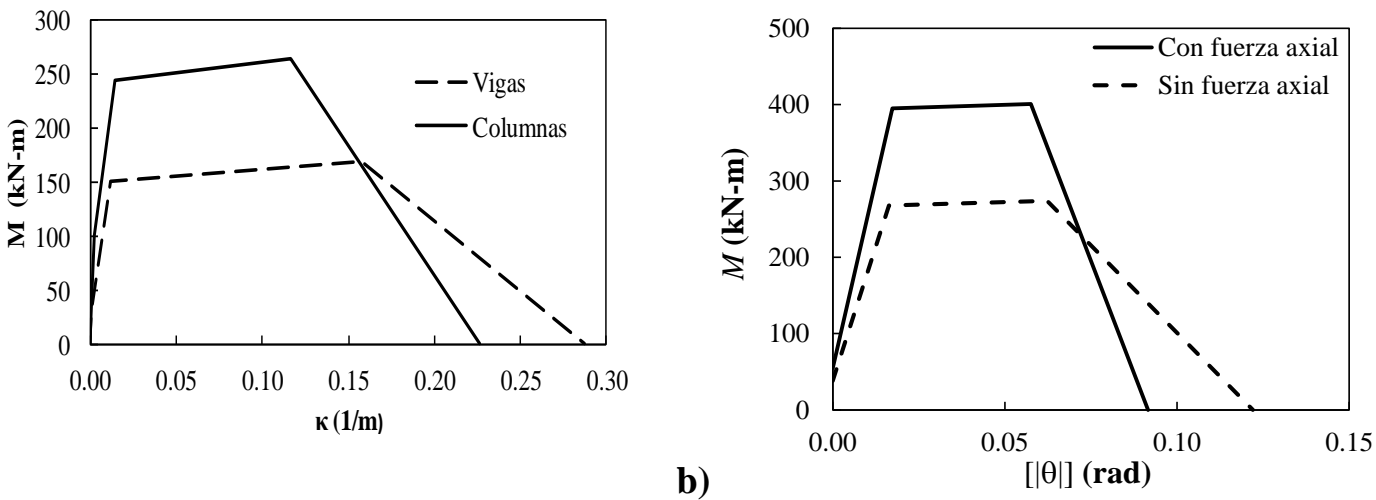

Figura 29. Comportamientos constitutivos: a) $M-\kappa$ y b) $M-\theta$ 

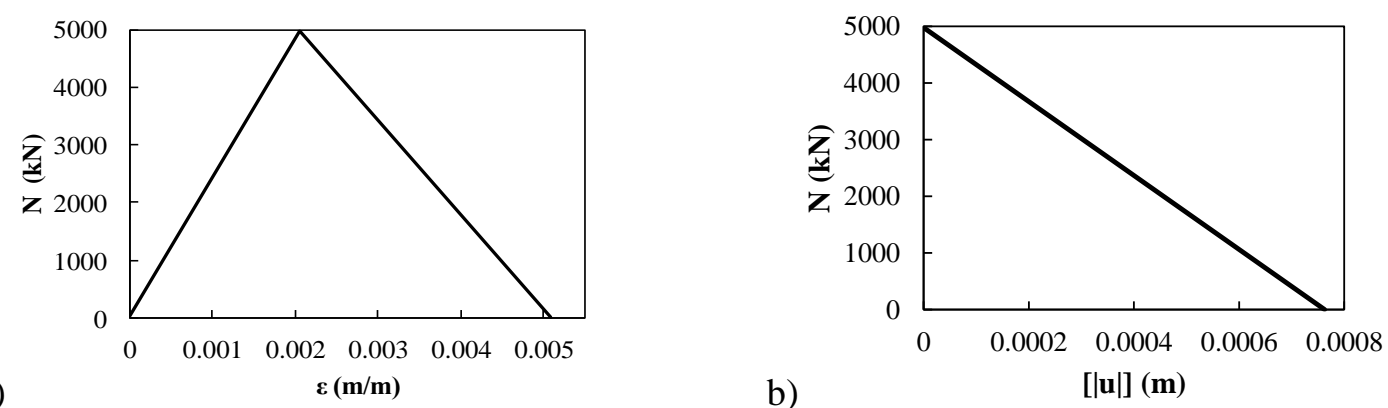

Figura 30. Comportamientos constitutivos: a) $N-\varepsilon$ y b) $N-[|\mathrm{u}|]$

Las vigas y las columnas del marco se discretizaron con elementos viga-columna delgada como se muestra en la Figura 27b. Es importante considerar que los elementos son capaces de capturar sólo una articulación por elemento, por lo que este marco requiere al menos de dos elementos para discretizar las vigas para capturar las dos articulaciones correspondientes. Para la solución numérica del marco, primeramente se aplicaron y se mantuvieron constantes las cargas verticales de $700 \mathrm{kN}$ en los nodos 5 y 6 , las cuales provocan fuerzas de compresión en las columnas. Posteriormente se impuso gradualmente un desplazamiento horizontal hacia la derecha en el nodo 5, cuya reacción corresponde a la magnitud de $F$. La Figura 31 muestra la curva calculada $F$ - $u$, la cual se compara con la solución experimental reportada por Vecchio y Emara (1992) y la solución numérica reportada por Jukic (2013), quien utilizó elementos vigas con discontinuidades en la rotación. En la rama ascendente, el comportamiento de las tres curvas es semejante; sin embargo, en la meseta existe una diferencia mayor entre la curva experimental y la obtenida por Jukic (2013), que con la curva obtenida con el elemento viga-columna con discontinuidades desarrollada en este trabajo.

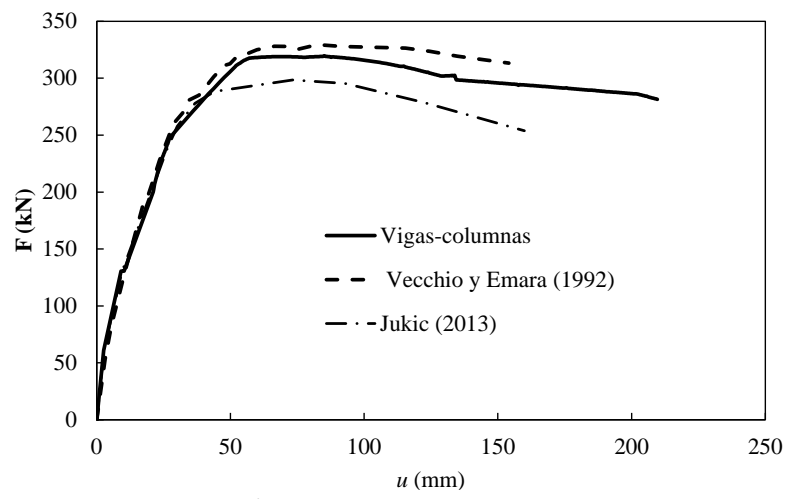

Figura 31. Curva $F-u$

La secuencia de formación de articulaciones se muestra en la Figura 32a, donde simultáneamente ocurren dos articulaciones en los extremos de la viga del primer nivel, posteriormente ocurren otras dos articulaciones en los extremos de la viga del segundo nivel. Después ocurre una articulación en la parte inferior de la columna derecha y, finalmente, ocurre una articulación en la parte inferior de la columna izquierda. En la Figura 32b se muestra la variación del momento contra el incremento de carga normalizado de los puntos donde ocurrió cada una de las articulaciones. 
a)

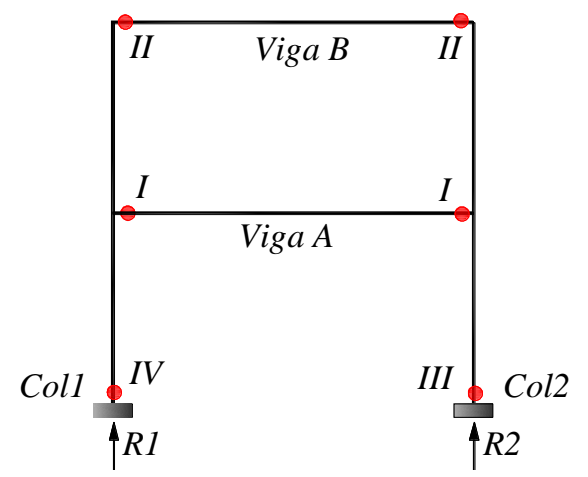

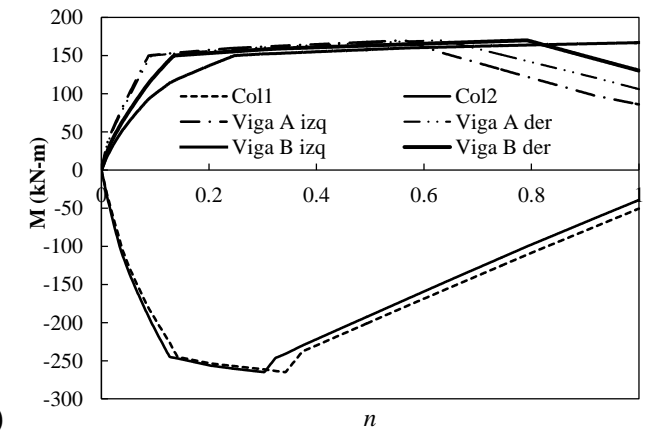

b)

Figura 32. Articulaciones: a) secuencia de ocurrencia y b) variación del momento

La Figura 33 muestra la variación de las magnitudes de las reacciones verticales $R_{l}$ y $R_{2}$ de los apoyos, donde se presentan las fuerzas axiales con mayor magnitud y donde se examinó la ocurrencia del daño por momento flexionante y fuerza normal. Como se esperaba, los elementos mecánicos en las columnas no presentaron fuerzas axiales en tensión durante la acción de la carga $F$, debido a la acción constante de las fuerzas a compresión aplicadas verticalmente en los nodos 5 y 6 . También se observa que la magnitud de fuerza a compresión máxima no excede la fuerza normal última de $N_{u}=5000 \mathrm{kN}$ mostrada en la Figura 33b.

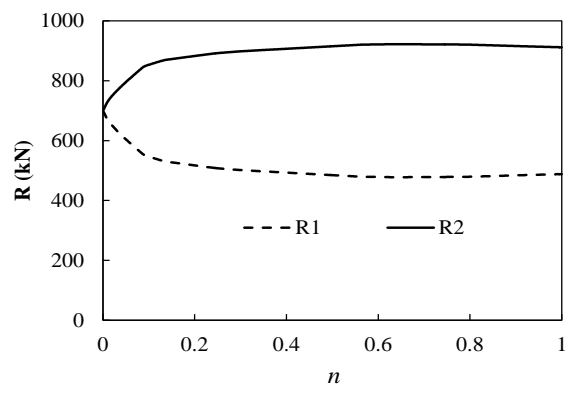

Figura 33. Variación de la magnitud las reacciones $R_{l}$ y $R_{2}$

\section{CONCLUSIONES}

En este trabajo se desarrolló un elemento viga-columna delgado, que tiene la capacidad de modelar discontinuidades en la rotación y en el desplazamiento axial y otro elemento grueso, que además es capaz de modelar la discontinuidad del desplazamiento transversal. Estos elementos se validan con ejemplos de aplicación numérica que muestran la capacidad de estos elementos para modelar el proceso de falla en estructuras. Una vez realizada la formulación, implantación y aplicación de los elementos finitos vigacolumna se concluye lo siguiente:

- Las matrices de rigideces de los elementos finitos viga-columna con discontinuidades interiores son simétricas, lo que reduce el tiempo de cómputo y evita posibles problemas de inestabilidad numérica que pueden presentar otras aproximaciones.

- Los modelos constitutivos de daño permiten modelar el comportamiento del elemento desde su intervalo elástico, inicio y evolución de las discontinuidades hasta el colapso total.

- Los elementos finitos viga-columna con discontinuidades interiores desarrollados no sólo se aplican a estructuras de concreto reforzado, pues para aplicarlos a otros materiales basta con modificar las propiedades mecánicas de los modelos constitutivos. 
- $\quad$ El elemento finito viga-columna delgado es capaz de modelar adecuadamente discontinuidades de desplazamiento axial y rotación, pero el elemento finito viga-columna grueso además puede modelar el desplazamiento transversal.

- Se demostró que el trabajo que se induce a una estructura se disipa adecuadamente mediante la liberación de energía en la formación de articulaciones embebidas en los elementos viga-columna desarrollados.

- La solución obtenida con los elementos desarrollados son independientes de la malla, pues se obtuvieron los mismos resultados con distintas discretizaciones. Sin embargo, es importante considerar que los elementos son capaces de capturar sólo una articulación por elemento, por lo que el último ejemplo del marco requiere al menos de dos elementos para discretizar las vigas para capturar las dos articulaciones correspondientes.

- Estos elementos se utilizarán en trabajos futuros para obtener la curva de capacidad de marcos, edificios y de otro tipo de estructuras como túneles, los cuales se pueden modelar con elementos vigacolumna.

\section{AGRADECIMIENTOS}

El primer autor agradece al proyecto "182736 Análisis y diseño de losas de concreto" auspiciado por la Secretaría de Educación Pública y el CONACyT. El segundo autor agradece la beca de estudios de Doctorado al CONACYT y a la Universidad Tecnológica de Panamá por las facilidades otorgadas. Ambos autores agradecen a la Universidad Autónoma Metropolitana por las facilidades proporcionadas a la realización de este y por el patrocinio al proyecto titulado "Análisis de losas de concreto e implantación de elementos finitos con discontinuidades embebidas".

\section{REFERENCIAS}

Armero, F y D Ehrlich (2006), "Numerical modeling of softening hinges in thin Euler-Bernoulli beams", Computer \& Structures, Vol. 84, pp. 641-656. DOI:10.1016/j.compstruc.2005.11.010

Baker, J B y J Hyman (1969), Plastic design of frames, Cambridge University Press, Vols. I y II.

Dujc, J, B Brank y A Ibrahimbegovic (2010), "Multi-scale computational model for failure analysis of metal frames that includes softening and local buckling", Computer Methods in Applied Mechanics and Engineering, Vol. 199, pp. 1371-1385. DOI:10.1016/j.cma.2009.09.003

Ehrlich, D y F Armero (2004), "Finite element methods for the analysis of softening plastic hinge in beams and frames", Computer and Mechanics, Vol. 35, pp. 237-264. DOI: 10.1007/s00466-004-0575-z

Juárez, G (2006), "Modelado numérico de problemas de fractura en sólidos mediante discontinuidades interiores”, Tesis de Doctorado, Universidad Nacional Autónoma de México, México.

Juárez, G y A G Ayala (2012), "Finite element variational formulation for beams with discontinuities", Finite Elements in Analysis and Design, Vol. 54, pp. 37-47. DOI:10.1016/j.finel.2012.01.004

Jukic, M (2013) "Finite element for modeling of localized failure in reinforced concrete", Tesis de Doctorado, Ljubljana, Univerza v Ljubljana, Slovenia.

Jukic, M, B Brank y A Ibrahimbegovic (2013) "Embedded discontinuity finite element formulation for failure analysis of planar reinforced concrete beams and frames", Engineering Structures, 50,115125. DOI:10.1016/j.engstruct.2012.07.028 
Oliver, J (1996), "Modelling strong discontinuities in solid mechanics via strain softening constitutive equations, Part 1: Fundamentals", Vol. 39, No. 21, pp. 3575-3600. DOI: 10.1002/(SICI)10970207(19961115)39:21<3575::AID-NME65>3.0.CO;2-E. Part 2: "Numerical simulation", International Journal for Numerical Methods in Engineering, Vol. 39, No. 21, pp. 3601-3623. DOI: 10.1002/(SICI)1097-0207(19961115)39:21<3601::AID-NME64>3.0.CO;2-4

Tena, A (2007), Análisis de estructuras con métodos matriciales, Editorial Limusa, $1^{\text {a }}$ edición, 559 p., México.

Taylor, L R (2008), “A finite element analysis program (FEAP) v8.2”, Department of Civil and Environmental Engineering, University of California at Berkeley, Berkeley, CA.

Vecchio, F J y M B Emara (1992), "Shear deformations in reinforced concrete frames", ACI Structural Journal, Vol. 89, No. 1, pp. 46-56. 